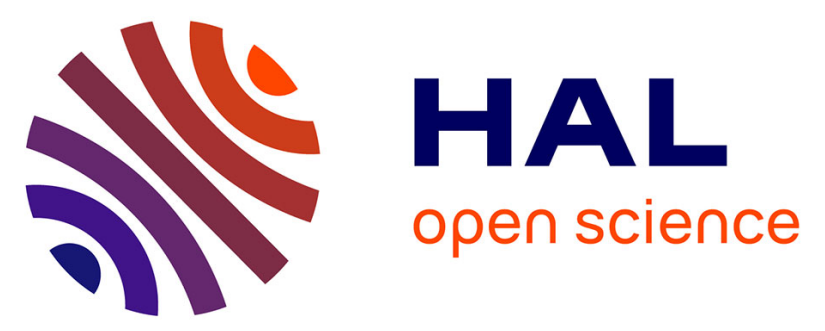

\title{
Tokamak research at the Ioffe Institute
}

N N Bakharev, G I Abdullina, V I Afanasyev, A B Altukhov, L G Askinazi, N A Babinov, A N Bazhenov, A A Belokurov, M D Blekhshtein, E N Bondarchuk, et al.

\section{To cite this version:}

N N Bakharev, G I Abdullina, V I Afanasyev, A B Altukhov, L G Askinazi, et al.. Tokamak research at the Ioffe Institute. Nuclear Fusion, 2019, 59, pp.112022. 10.1088/1741-4326/ab22dc . hal-02434462

\section{HAL Id: hal-02434462 \\ https://hal.univ-lorraine.fr/hal-02434462}

Submitted on 10 Jan 2020

HAL is a multi-disciplinary open access archive for the deposit and dissemination of scientific research documents, whether they are published or not. The documents may come from teaching and research institutions in France or abroad, or from public or private research centers.
L'archive ouverte pluridisciplinaire HAL, est destinée au dépôt et à la diffusion de documents scientifiques de niveau recherche, publiés ou non, émanant des établissements d'enseignement et de recherche français ou étrangers, des laboratoires publics ou privés. 


\title{
Tokamak research at the loffe Institute
}

\author{
N.N. Bakharev ${ }^{1}$, G.I. Abdullina ${ }^{1}$, V.I. Afanasyev ${ }^{1}$, A.B. Altukhov ${ }^{1}$, \\ L.G. Askinazi ${ }^{1}$, N.A. Babinov ${ }^{1}$, A.N. Bazhenov ${ }^{1}$, A.A. Belokurov ${ }^{1}$,
}

M.D. Blekhshtein ${ }^{2}$, E.N. Bondarchuk ${ }^{3}$, I.M. Bukreev ${ }^{1}$, V.V. Bulanin ${ }^{1,2}$, An.P. Chernakov ${ }^{4}$, F.V. Chernyshev ${ }^{1}$, I.N. Chugunov ${ }^{1}$, A.M. Dmitriev ${ }^{1}$, D.N. Doinikov ${ }^{1}$, V.V. Dyachenko ${ }^{1}$, L.A. Esipovv ${ }^{1}$, D.B. Gin ${ }^{1}$, A.V. Gorbunov ${ }^{5}$, A.D. Gurchenko ${ }^{1}$, E.Z. Gusakov ${ }^{1}$, V.K. Gusev ${ }^{1}$, S. Heuraux ${ }^{6}$, M.V. Iliasova ${ }^{1}$, M.A. Irzak' , S.N. Kamenshikov ${ }^{3}$, A.A. Kavin ${ }^{3}$, E.M. Khilkevitch ${ }^{1}$, N.A. Khromov ${ }^{1}$, E.O. Kiselev ${ }^{1}$, T.P. Kiviniemi ${ }^{7}$, A.A. Kobelev ${ }^{2}$, V.A. Kornev ${ }^{1}$, A.N. Koval ${ }^{1}$, D.V. Kouprienko ${ }^{1}$, S.V. Krikunov ${ }^{1}$, O.L. Krutkin ${ }^{1}$, G.S. Kurskiev ${ }^{1}$, S.I. Lashkul ${ }^{1}$, S.V. Lebedev ${ }^{1}$, C. Lechte $^{8}$, S. Leerink ${ }^{7}$, A.E. Litvinov ${ }^{1}$, K.M. Lobanov ${ }^{3}$, S.V. Masyukevich ${ }^{1}$, A.A. Martynov ${ }^{9}$, S.Yu. Medvedev ${ }^{9}$, A.D. Melnik ${ }^{1}$, V.B. Minaev ${ }^{1}$, A.B. Mineev ${ }^{3}$, M.I. Mironov' ${ }^{1}$, I.V. Miroshnikov ${ }^{1}$, E.E. Mukhin ${ }^{1}$, V.O. Naidenov ${ }^{1}$, A.S. Navolotsky ${ }^{1}$, V.G. Nesenevich ${ }^{1}$, P. Niskala ${ }^{7}$, A.N. Novokhatskii ${ }^{1}$, K.Yu. Oshuev ${ }^{1}$, M.I. Patrov ${ }^{1}$, A.V. Petrov ${ }^{2}$, M.P. Petrov ${ }^{1}$, S.Ya. Petrov ${ }^{1}$, Yu.V. Petrov ${ }^{1}$, I.A. Polunovsky ${ }^{1}$, A.Yu. Popov ${ }^{1}$, A.G. Razdobarin ${ }^{1}$, D.V. Razumenko ${ }^{1}$, V.V. Rozhdestvensky ${ }^{1}$, N.V. Sakharov ${ }^{1}$, D.S. Samsonov ${ }^{1}$, A.N. Saveliev ${ }^{1}$, V.A. Senichenkov ${ }^{1}$, P.B. Shchegolev ${ }^{1}$, A.E. Shevelev ${ }^{1}$, A.D. Sladkomedova ${ }^{1}$, A.I. Smirnov ${ }^{1}$, A.S. Smirnov ${ }^{2}$, V.V. Solokha ${ }^{1}$, V.A. Solovei ${ }^{1}$, A.Yu. Stepanov ${ }^{1}$, A.Yu. Telnova ${ }^{1}$, V.A. Tokarev ${ }^{1}$, S.Yu. Tolstyakov ${ }^{1}$, P.V. Tretinnikov ${ }^{1}$, I.B. Tereschenko ${ }^{1}$, A.S. Tukachinsky ${ }^{1}$, E.A. Tukhmeneva ${ }^{1}$, V.I. Varfolomeev ${ }^{1}$, L.A. Varshavchick ${ }^{1}$, A.Yu. Yashin ${ }^{1,2}$, E.G. Zhilin ${ }^{10}$ and N.A. Zhubr ${ }^{1}$

1 Ioffe Institute, St. Petersburg 194021, Russian Federation

2 Peter the Great St. Petersburg Polytechnic University, St. Petersburg, Russian Federation

3 JSC «NIIEFA», St. Petersburg, Russian Federation

4 JSC «Spectral-Tech», St. Petersburg, Russian Federation

5 NRC Kurchatov Institute, Moscow, Russian Federation

${ }^{6}$ Institute Jean Lamour UMR 7198 CNRS, Université de Lorraine, 54000 Nancy, France

7 Aalto University, Espoo, Finland

${ }^{8}$ Institute of Interfacial Process Engineering and Plasma Technology, 70569 Stuttgart, Germany

9 Keldysh Institute of Applied Mathematics RAS, Moscow, Russian Federation

${ }^{10}$ Ioffe Fusion Technology Ltd, St. Petersburg, Russian Federation

E-mail: bakharev@mail.ioffe.ru

Published 30 August 2019

\section{Abstract}

Recent research at three small tokamaks with different parameters located at the Ioffe Institute - the spherical tokamak Globus-M, the large aspect ratio tokamak FT-2 and the compact tokamak TUMAN-3M- are reviewed. This overview covers energy confinement (Globus-M and FT-2), L-H transition (TUMAN-3M and FT-2), Alfvén waves (Globus-M and TUMAN-3M), ion cyclotron emission (TUMAN-3M), major plasma discharge disruption (Globus-M) and scrape-off layer (Globus-M) studies. A full-f global gyrokinetic modeling benchmark using synthetic diagnostics in FT-2 is described. Anomalous absorption and emission in electron cyclotron resonance heating experiments due to the parametric excitation of localized upper hybrid waves are analyzed theoretically. Progress in the development of the neutral particle analysis, gamma-ray spectrometry and divertor Thomson scattering combined 
with laser-induced fluorescence diagnostics for ITER is discussed. The status of the new Globus-M2 spherical tokamak is reported.

Keywords: energy confinement, Alfvén eigenmodes, SOL, gyrokinetic modeling, ICE, L$\mathrm{H}$ transition, disruption

\section{Introduction}

In 2018, the Ioffe Institute celebrated its 100th anniversary. Plasma physics and thermonuclear fusion research at the Ioffe Institute has been going on for more than 60 years. At present, the study of the various tokamak physics aspects is conducted on small tokamaks in a wide range of the experimental conditions: $R / a=1.6, B_{\mathrm{T}}=0.5 \mathrm{~T}, I_{\mathrm{p}}=250 \mathrm{kA}$ (Globus-M); $R / a=2.4, B_{\mathrm{T}}=1.0 \mathrm{~T}, I_{\mathrm{p}}=180 \mathrm{kA}(\mathrm{TUMAN}-3 \mathrm{M}) ; R / a=7.0$, $B_{\mathrm{T}}=3.0 \mathrm{~T}, I_{\mathrm{p}}=40 \mathrm{kA}(\mathrm{FT}-2)$. In addition, there is cooperation with other tokamaks all over the world, and three ITER diagnostics - neutral particle analysis, gamma-ray spectrometry and divertor Thomson scattering (DTS) combined with laser-induced fluorescence (LIF) — are being developed. In this overview recent results of the tokamak research at the Ioffe Institute are described.

Globus M [1, 2], which has recently been replaced by a new Globus-M2, was a compact spherical tokamak (ST) $(R \approx 36 \mathrm{~cm}$, aspect ratio $A \approx 1.5$, elongation $k<2$ ) equipped with neutral beam injection (NBI), ion cyclotron resonance (ICR) heating and lower hybrid current drive (LHCD) systems. Its distinguishing features were a high normalized Larmor radius and collisionality, high heating power density and a small plasma-wall distance. In this paper the main results, dedicated to the study of energy confinement, Alfvén eigenmodes, discharge disruption and scrape-off layer (SOL) study are described. TUMAN-3M is a compact tokamak with $R=55 \mathrm{~cm}, a=22 \mathrm{~cm}$, equipped with the neutral beam injector. The main results of the investigation in Tuman-3M of the high frequency magnetohydrodynamic oscillations in the ion cyclotron and Alfvén frequency ranges and the study of the $\mathrm{L}-\mathrm{H}$ transition, initiated by geodesic acoustic mode (GAM) [3] activity and pellet injection, are described. FT-2 is a large aspect ratio machine $(R=55 \mathrm{~cm}, a=8 \mathrm{~cm}$, $\left.I_{\mathrm{p}}=40 \mathrm{kA}\right)$, equipped with the lower hybrid $(\mathrm{LH})$ heating system $\left(f_{0}=920 \mathrm{MHz}\right)$ for study of LHCD at low density and LH heating of ions at higher plasma densities [4]. FT-2 is equipped with the unique complex of microwave backscattering diagnostics. Its discharges are characterized by a good reproducibility and therefore FT-2 is used for research in different areas. In this overview the main results of the energy confinement study in hydrogen and deuterium Ohmically heated $(\mathrm{OH})$ plasma and full-f global gyrokinetic modeling benchmark using synthetic diagnostics are described.

The paper is organized as follows. In section 2, energy confinement in the NBI-heated discharges of the Globus-M $\mathrm{ST}$ and in the $\mathrm{OH}$ discharges of the large aspect ratio FT-2 tokamak are described. In section $3, \mathrm{~L}-\mathrm{H}$ transitions in
TUMAN-3M, initiated by GAM activity and pellet injection, are studied. Toroidal Alfvén eigenmodes in Globus-M NBIheated discharges and Alfvén-type activity in TUMAN-3M $\mathrm{OH}$ plasma are described in section 4. Ion cyclotron emission in $\mathrm{OH}$ and NBI-heated TUMAN-3M plasma is discussed in section 5. Section 6 is devoted to the discharge disruption investigation in Globus-M, followed by the SOL study in section 7. Benchmarking of the full-f global gyrokinetic modeling results against the FT-2 tokamak Doppler reflectometry (DR) data using synthetic diagnostics is presented in section 8 . In section 9,anomalous absorption and emission in electron cyclotron resonance heating $(\mathrm{ECRH})$ experiments due to the parametric excitation of localized upper hybrid (UH) waves is described. The ITER neutral particle analyzer, gammaray spectrometer and DTS system, combined with LIF, are reviewed in section 10. The status of the new Globus-M2 machine is presented in section 11. Finally, a short summary is given in section 12 .

\section{Energy confinement}

\subsection{Energy confinement in the Globus-M spherical tokamak}

Thermal energy confinement in STs is one of the key points under investigation, because it significantly differs from the confinement in conventional machines. Relatively low toroidal magnetic fields in STs, together with their good confinement capabilities allow stable operation at significantly high $\beta$ (the ratio of plasma pressure to magnetic field pressure). High $\beta$, a large normalized ion gyroradius $\left(\rho^{*}\right)$, a high fraction of trapped particles and strong plasma shaping lead to essential differences in plasma turbulence that determine particle and heat fluxes. The results, obtained on MAST [5] and NSTX [6], have shown the difference between the engineering scaling for energy confinement time $\left(\tau_{\mathrm{E}}\right)$ in STs and the conventional IPB98 $(y, 2)[7]$. The main distinction is a strong dependence of $\tau_{\mathrm{E}}$ on the toroidal magnetic field in STs, which is negligible in conventional machines. It is connected with much stronger electron heat diffusivity dependence on collisionality. Further experiments and analysis have confirmed this statement $[8,9]$.

The Globus-M ST operated in the same range of the toroidal magnetic fields as MAST and NSTX, however other engineering parameters differed significantly $(R \approx 36 \mathrm{~cm}$, aspect ratio $A \approx 1.5$, elongation $k<2$ ). In contrast to the previous $\mathrm{OH}$ experiments $[10,11]$, energy confinement was investigated in NBI-heated ELMy H-mode deuterium plasma using 26-28 keV $0.35-0.75 \mathrm{MW}$ deuterium beams [12]. The range of the major plasma parameters was as follows: 


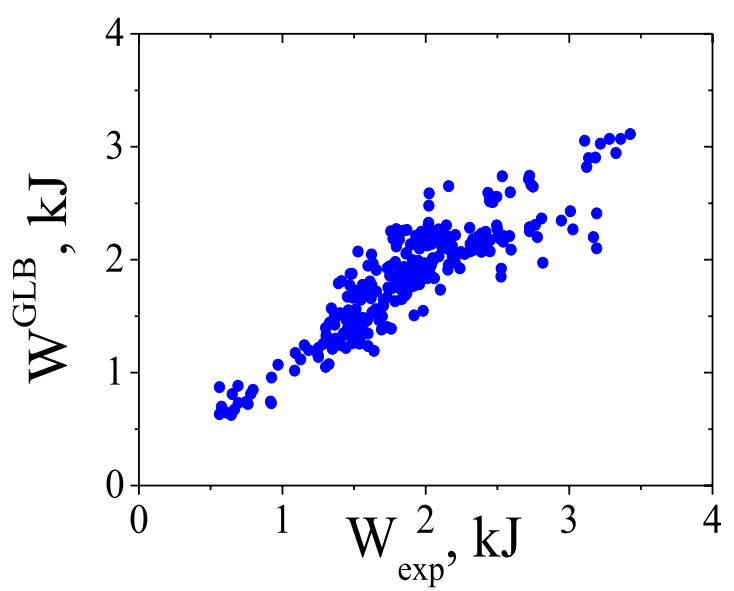

Figure 1. The comparison of the plasma stored energy with $\tau_{\mathrm{E}}^{\mathrm{GLB}} \sim I_{\mathrm{p}}^{0.51 \pm 0.26} B_{\mathrm{T}}^{1.2 \pm 0.1} P_{\text {abs }}^{-0.54 \pm 0.26} n_{\mathrm{e}}^{0.67 \pm 0.04}$ regression fit.

$I_{\mathrm{p}}=0.12-0.25$ MA, $B_{\mathrm{T}}=0.25-0.5 \mathrm{~T}$, absorbed heating power $P_{\text {abs }}=0.2-0.8 \mathrm{MW}$, and averaged electron density $n_{\mathrm{e}}=1.8-5.5 \times 10^{19} \mathrm{~m}^{-3}$. It was found that $\operatorname{IPB} 98(y, 2)$ functional dependence is poorly suited for describing the experiment. The $\mathrm{H}$-factor was in the range of $0.5-1.3$ with a root mean square error (RMSE) $>60 \%$ [13]. The regression analysis of the database yields following results: $\tau_{\mathrm{E}}^{\mathrm{GLB}} \sim I_{\mathrm{p}}^{0.51 \pm 0.26} B_{\mathrm{T}}^{1.2 \pm 0.1} P_{\mathrm{abs}}^{-0.54 \pm 0.26} n_{\mathrm{e}}^{0.67 \pm 0.04}$ with $\mathrm{RMSE}=$ $25 \%$ (figure 1). The confinement time dependence on the toroidal magnetic field is very strong, which is consistent with the ST trend $[5,6]$. One of the most important points is the dependence of the dimensionless energy confinement time $\left(B_{\mathrm{T}} \tau_{\mathrm{E}}\right)$ on collisionality $\nu^{*} \sim n_{\mathrm{e}} / T^{2}$. A fusion reactor or fusion neutron source will operate in the range of sufficiently lower collisionality, and therefore extrapolation of the experimental data to the lower $\nu^{*}$ values is of great interest. It was found that for conventional tokamaks, thermal energy confinement has a negligible dependence on collisionality and ITER scaling predicts $B_{\mathrm{T}} \tau_{\mathrm{E}} \sim \beta_{\mathrm{T}}^{-0.9} \nu^{*-0.01} q_{\text {eng }}^{-3}$ [7], however a different trend is observed for STs. The main feature of the thermal energy confinement in an ST is a strong $\tau_{\mathrm{E}}$ dependence on collisionality $[8,9]$. For the MAST ELMy H-mode $B_{\mathrm{T}} \tau_{\mathrm{E}} \sim$ $q^{-0.85 \pm 0.2} \nu^{*-0.82 \pm 0.1}$, for the NSTX ELMy H-mode: $B_{\mathrm{T}} \tau_{\mathrm{E}} \sim$ $\nu^{*-0.79 \pm 0.1}$. A formal regression fit of the Globus-M data in dimensionless space yields $B_{\mathrm{T}} \tau_{\mathrm{E}} \sim \rho^{*-2.7} \beta_{\mathrm{T}}^{1.45} \nu^{*-0.45} q_{\mathrm{eng}}^{0.85}$ with $\mathrm{RMSE}=10 \%$. To verify the result the dedicated collisionality scan was performed while the other dimensionless parameters were kept constant. For this purpose we have used discharges with $B_{\mathrm{T}}=0.32,0.4,0.5 \mathrm{~T}$ and $I_{\mathrm{p}}=0.15,0.2$, 0.25 MA. The exponent indexes calculation, performed by log-linear regression, yields the dependence $B \tau_{\mathrm{E}} \sim \nu^{*-0.4 \pm 0.2}$ (figure 2). The dependence on $\nu^{*}$ is even stronger if the variation of $\rho^{*}$ is taken into account through the Bohm and gyroBohm assumptions, with the normalized confinement going as $\rho_{*}^{2} B \tau_{\mathrm{E}} \sim \nu^{*-0.45}$ and $\rho_{*}^{3} B \tau_{\mathrm{E}} \sim \nu^{*-0.52}$, respectively. As a result the confinement time dependence on collisionality can be roughly limited to values $B_{\mathrm{T}} \tau_{\mathrm{E}} \sim \nu^{-0.46 \pm 0.5}$ in the collsionality range $\nu^{*}=0.02-0.4$. This dependence differs from the one in NSTX and MAST but it is significantly stronger than dependence, predicted by ITER scaling [7]. The main

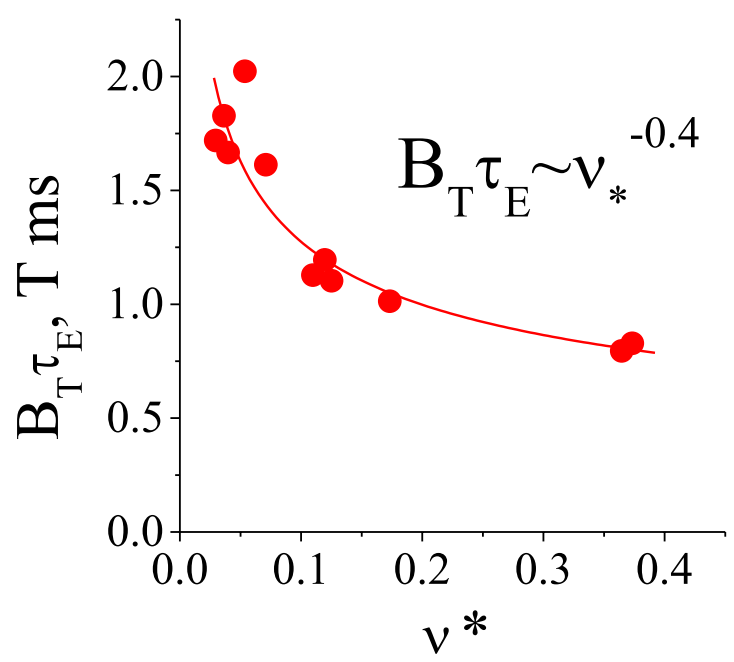

Figure 2. The dependence of normalized confinement time on collisionality.

reason for confinement improvement is the decrease of the electron heat conductivity. Transport analysis using ASTRA code have shown that $B / \chi_{\mathrm{e}} \sim \nu^{*-0.4 \pm 0.2}$. The result is consistent with the obtained energy confinement time dependence on collisionality.

\subsection{Energy confinement in the large aspect ratio FT-2 tokamak}

A clear isotope effect is found in high density regimes (HDRs) both for energy and particle channels [14] in FT-2 experiments. HDR Ohmic discharges were performed in two different working gases: hydrogen and deuterium. Figure 3 shows the energy confinement time dependence on the line-averaged density. No visible isotope effect on global energy confinement is observed at densities up to $\left\langle n_{\mathrm{e}}\right\rangle \sim 5 \times 10^{19} \mathrm{~m}^{-3}$, presenting typical linear Ohmic confinement (LOC) [15] behavior.

For higher densities hydrogen plasma demonstrates saturation of $\tau_{\mathrm{E}}\left(n_{\mathrm{e}}\right)$ linear dependency which can be interpreted as transition to the saturated Ohmic confinement (SOC) [15] regime (LOC-SOC transition), however $\tau_{\mathrm{E}}$ in deuterium grows faster than in the LOC stage. The energy confinement time became twice as high in deuterium than in hydrogen at $\left\langle n_{\mathrm{e}}\right\rangle \sim(8-9) \times 10^{19} \mathrm{~m}^{-3}$. The particle confinement in D plasma possesses features of the H-mode: a strong $\mathrm{D}_{\beta}$ line emission drop, growth of peripheral density gradient and suppression of turbulence at the plasma edge. The explanation of the isotopic difference in the improved Ohmic confinement [15] onset, and consequently in transport, is based on the relation between radial electric field shear and turbulence growth rate, calculated with gyrokinetic simulation and using analytical estimations.

\section{L-H transition}

Experiments in TUMAN-3M show that in low density discharges $\left(\left\langle n_{\mathrm{e}}\right\rangle=0.5-1.2 \times 10^{19} \mathrm{~m}^{-3}\right)$, the L-H transition is present only if a strong external sheared $E_{\mathrm{r}}$ is applied. Two 


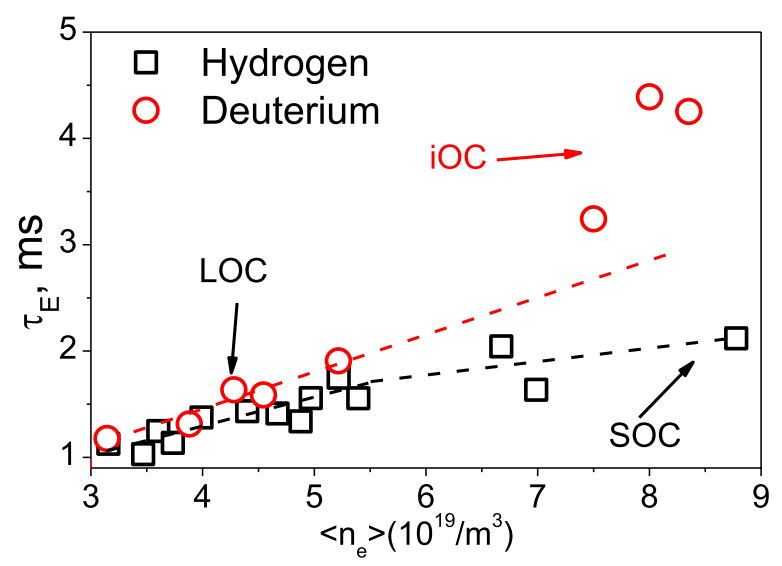

Figure 3. Energy confinement time dependence on chord-averaged density in hydrogen (squares) and deuterium (circles) plasmas.
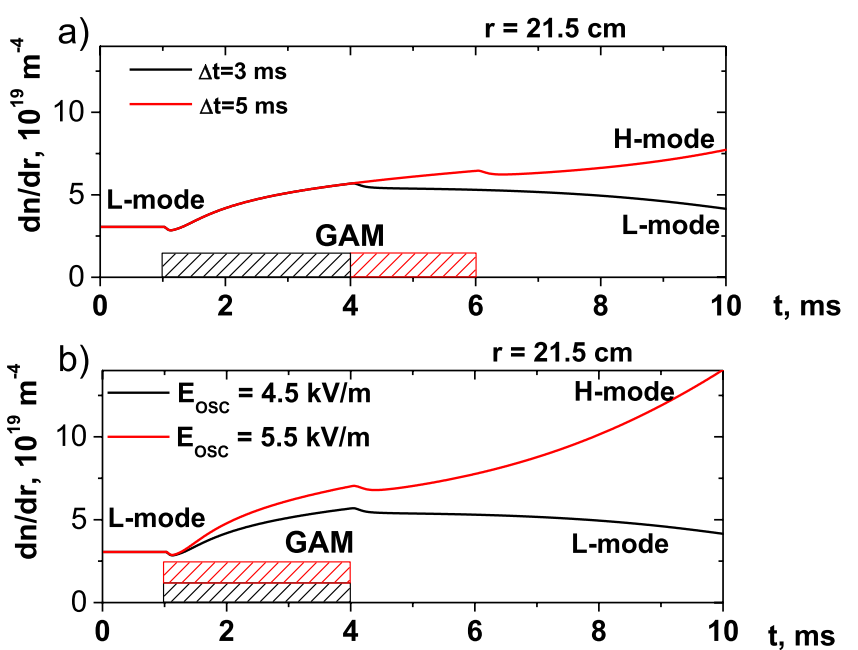

Figure 4. GAM in TUMAN-3M. GAM burst of sub-threshold duration $(a)$ or amplitude $(b)$ leads to $\mathrm{L}-\mathrm{H}$ transition initiation.

scenarios are discussed: discharge with electric field perturbations due to the strong geodesic acoustic mode (GAM) oscillations (in this scenario the $\mathrm{L}-\mathrm{H}$ transition is observed after a GAM burst in TUMAN-3M, while in low density discharges in FT-2 $\left(n(0)<5 \times 10^{19} \mathrm{~m}^{-3}\right)$ with stronger GAM activity it is absent); and cryogenic pellet injection in TUMAN-3M, which creates strong density and temperature gradients and, therefore, radial electric field gradient, and could lead to the $\mathrm{L}-\mathrm{H}$ transition, but does not guarantee it. Both scenarios were considered using the model of particle density profile evolution accounting for diffusion coefficient dependence on $E_{\mathrm{r}}$ shear $[16,17]$. Transport quantities and turbulence parameters were provided by the ELMFIRE gyrokinetic code. The modeling results have shown satisfactory agreement with the experiments: (1) in TUMAN-3M the GAM parameters threshold for $\mathrm{L}-\mathrm{H}$ transition is determined (figure 4), however for FT-2 the transition was not possible for experimental GAM parameter sets [18]; (2) in the case of the pellet injection, $\mathrm{L}-\mathrm{H}$ transition occurred if a part of the pellet was disintegrated in the pelletguide, resulting in a gas/'snow' puff so that the pellet ablation is peripheral; on the contrary, if the pellet is evaporated deeper in the plasma, there is no $\mathrm{L}-\mathrm{H}$ transition [19].

Results of the modeling proved the idea about the importance of the particle source for determining $\mathrm{L}-\mathrm{H}$ transition

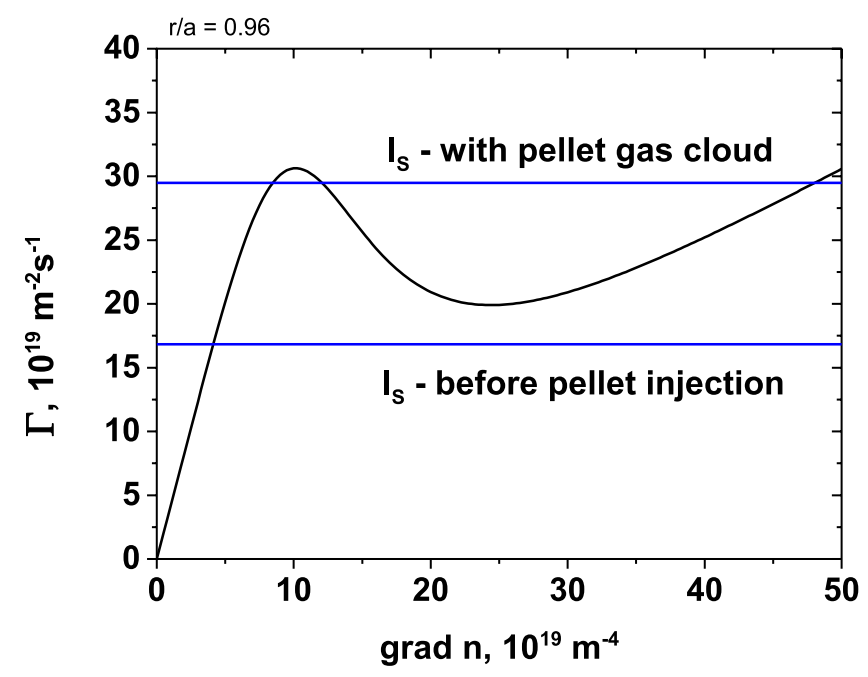

Figure 5. Nonlinear flux curve $\Gamma$ ( $\nabla n$ for TUMAN-3M before pellet injection and after peripheral pellet ablation, here the horizontal line indicates the integral source value (right side of equation (1)), the $N$-curve is the left side of equation (1), intersections show possible stationary solutions. The gas cloud additional source makes the existence of two solutions possible.

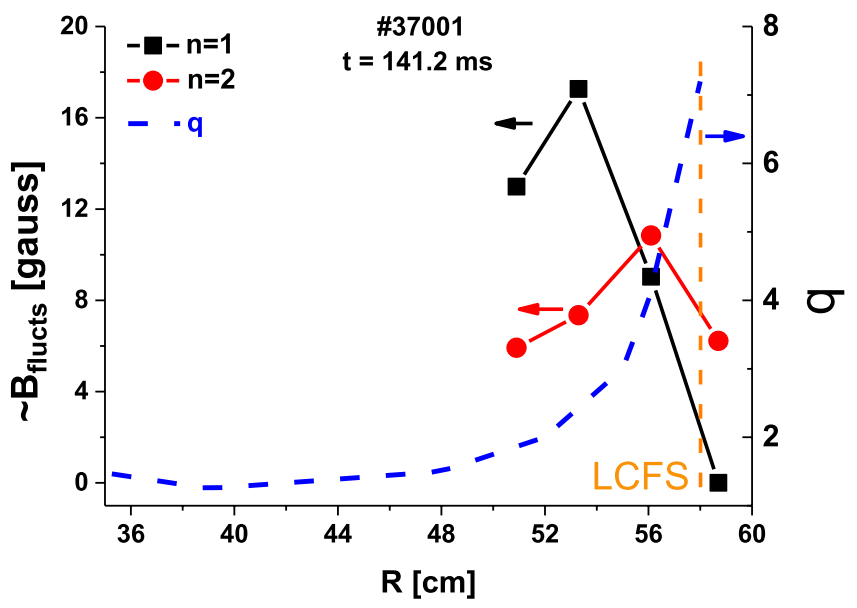

Figure 6. Localization of the TAE modes, measured with DBS, and $q$ profile in shot \#37001, $141.2 \mathrm{~ms}$.

possibility. This role is quite similar to the role of the ion heat source (heating power threshold) — if the particle source is too low for a certain scenario, a self-sustaining $\mathrm{H}$-mode is not possible. Particle source thresholds could be determined based on the diffusion coefficient dependence on $E_{\mathrm{r}}$ shear (and thus $\nabla n$ ). The analysis of non-linear particle flux dependence on density gradient:

$$
-D_{\text {eff }}\left(\frac{\partial n(r)}{\partial r}\right) \cdot \frac{\partial n(r)}{\partial r}=I_{\mathrm{S}}(r),
$$

where $D_{\text {eff }}$ is the effective diffusion coefficient and $I_{\mathrm{S}}$ the integral particle source, helps to reveal possible causes of $\mathrm{L}-\mathrm{H}$ transition presence or absence. Due to non-linearity, a diffusion equation in the stationary case has either two stable solutions (for the L- and H-mode), or one, for one confinement mode only, depending on the integral particle source value compared to the particle flux (see figure 5 , where the graphical solutions of equation (1) are shown for the case of 

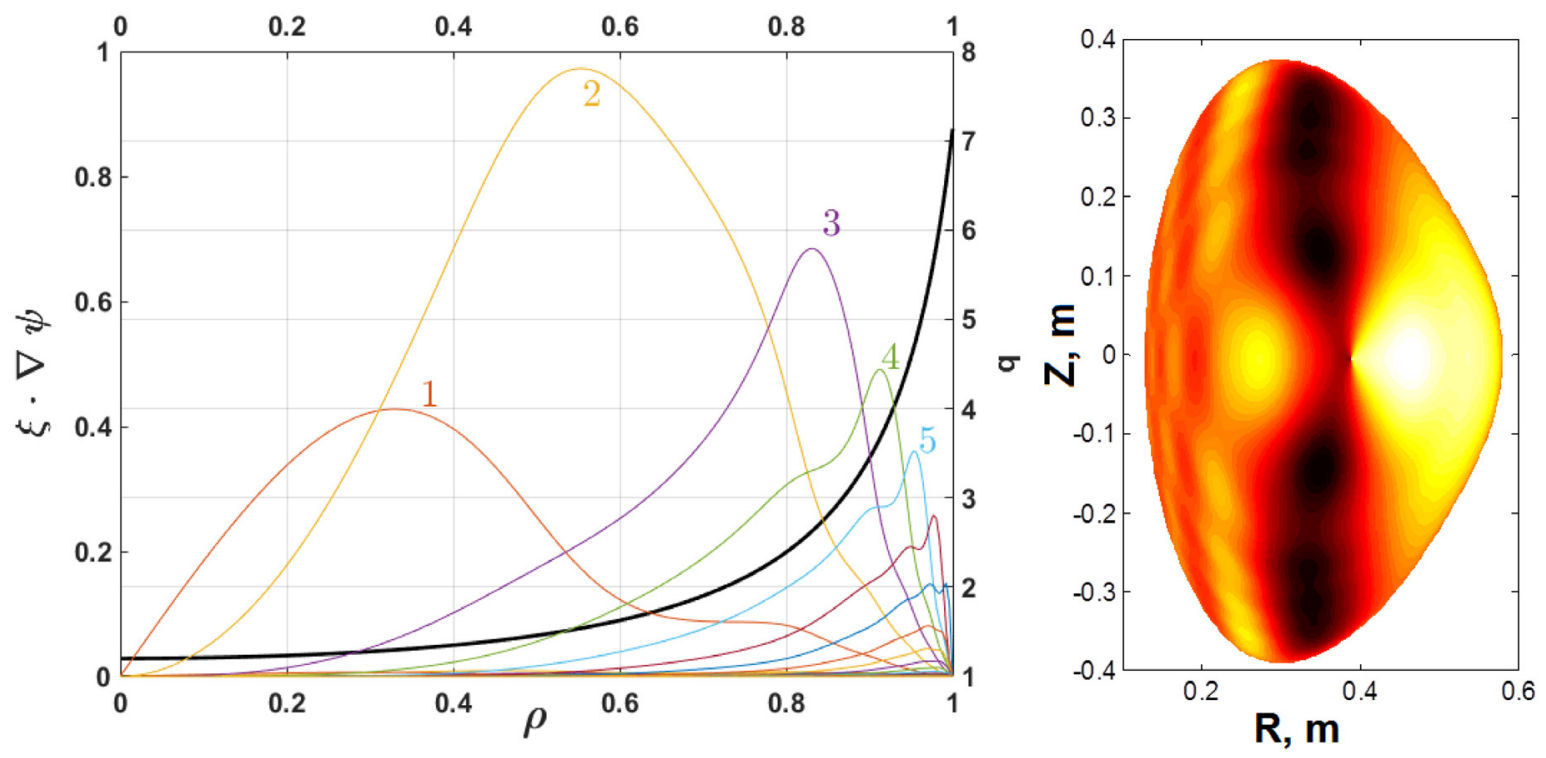

Figure 7. The structure of the $n=1$ TAE mode with a fixed boundary condition in the Alfvén continuum gap, frequency $155 \mathrm{kHz}$. Harmonics in straight field line coordinates and level lines of normal plasma displacement are shown.

TUMAN-3M pellet-injection experiments; the curve shows the dependence of particle flux on the density gradient, horizontal lines are integral source values). The case when the second stable solution (H-mode) is impossible corresponds to FT-2 low density discharge parameters or TUMAN-3M deep pellet injection scenarios.

\section{Alfvén waves}

\subsection{NBI-induced toroidal Alfvén eigenmodes}

Existence of fast particles with velocities in excess of the Alfvén velocity in plasma will lead to excitation of Alfvén instabilities that can provoke fast particle radial transport and losses. Investigation of the toroidal Alfvén eigenmodes (TAE) identified in earlier experiments with NBI heating on Globus-M [20, 21] were continued at the increased magnetic field and plasma current values of $0.5 \mathrm{~T}$ and $240 \mathrm{kA}$, respectively. We observed that the TAE-induced particle losses per burst of equal amplitude decrease with the increase of $B_{\mathrm{T}}$ and $I_{\mathrm{p}}$ [22]. The plasma current increase plays a decisive role in reduction of the losses, probably due to a decrease of the fast particle Larmor radius in the poloidal magnetic field [23]. The toroidal magnetic field increase gives a weaker effect, however $B_{\mathrm{T}}$ and $I_{\mathrm{p}}$ should be increased simultaneously to conserve the safety factor value. The obtained dependence is promising for a compact fusion neutron source on the base of a spherical tokamak, but should be checked in a wider parameter range, which is one of the tasks for the new Globus-M2 tokamak.

During the last experimental campaign on Globus-M, progress was reached in the investigation of the TAE structure and localization. The multichannel Doppler backscattering reflectometry (DBS) was successfully applied for this purpose. As TAEs perturb local magnetic and electrical fields, their influence on the plasma poloidal $E \times B$ rotational velocity could be detected from DBS measurements. The description of the method can be found in [24] and its first application on Globus-M in [25]. Multichannel probing at frequencies of 20, 29, 39 and $48 \mathrm{GHz}$ was applied. It allowed us to observe the TAE fluctuations at four spatially resolved points simultaneously. Figure 6 demonstrates the radial profiles of the magnetic fluctuation amplitudes obtained by the described method for the TAE modes $n=1$ and $n=2$ at $141.2 \mathrm{~ms}$ of shot \#37001. The dashed line shows the $q$ profile. It was obtained from the EFIT code calculations without plasma current profile measurements. We can conclude that TAEs are localized at the periphery of the plasma column, in the region of the normalized minor radii $\rho$ from $0.6-1$ and that modes with different $n$ have different localization. Modeling of the Alfvén continuum and mode structure for the TAE burst with profiles shown in figure 7 were performed with the modified KINX and CAXE codes [26]. Several global $n=1$ TAEs were found in the Alfvén continuum gap (the specific heat ratio $\Gamma=0$ ) assuming a fixed boundary condition. One of them, shown in figure 7 , is broadly consistent with the experimental data in terms of mode localization, structure and frequency.

\subsection{Alfvén waves (AWs) in $\mathrm{OH}$ plasma}

Lower-frequency AW-type oscillations are observed in TUMAN-3M at low densities $\overline{n_{\mathrm{e}}}<3 \times 10^{19} \mathrm{~m}^{-3}$ in both Ohmic and NBI regimes [27, 28] They exhibit a clear Alfvénic dependence on toroidal field and plasma density. Comparison of the experimentally measured frequency evolution with one, calculated using the local plasma density in a discharge with strong perturbation of density profile caused by $\mathrm{L}-\mathrm{H}$ transition, indicates that AWs are localized in the central region $r / a<0.5$ (figure 8(a)) [28, 29] and thus may be identified as a global Alfvén eigenmode rather than a TAE. AW activity in the TUMAN-3M plasma is usually represented by two types of oscillations: short bursts correlated with sawtooth crashes, and longer ones in between the crashes [30], see figure $8(b)$. 

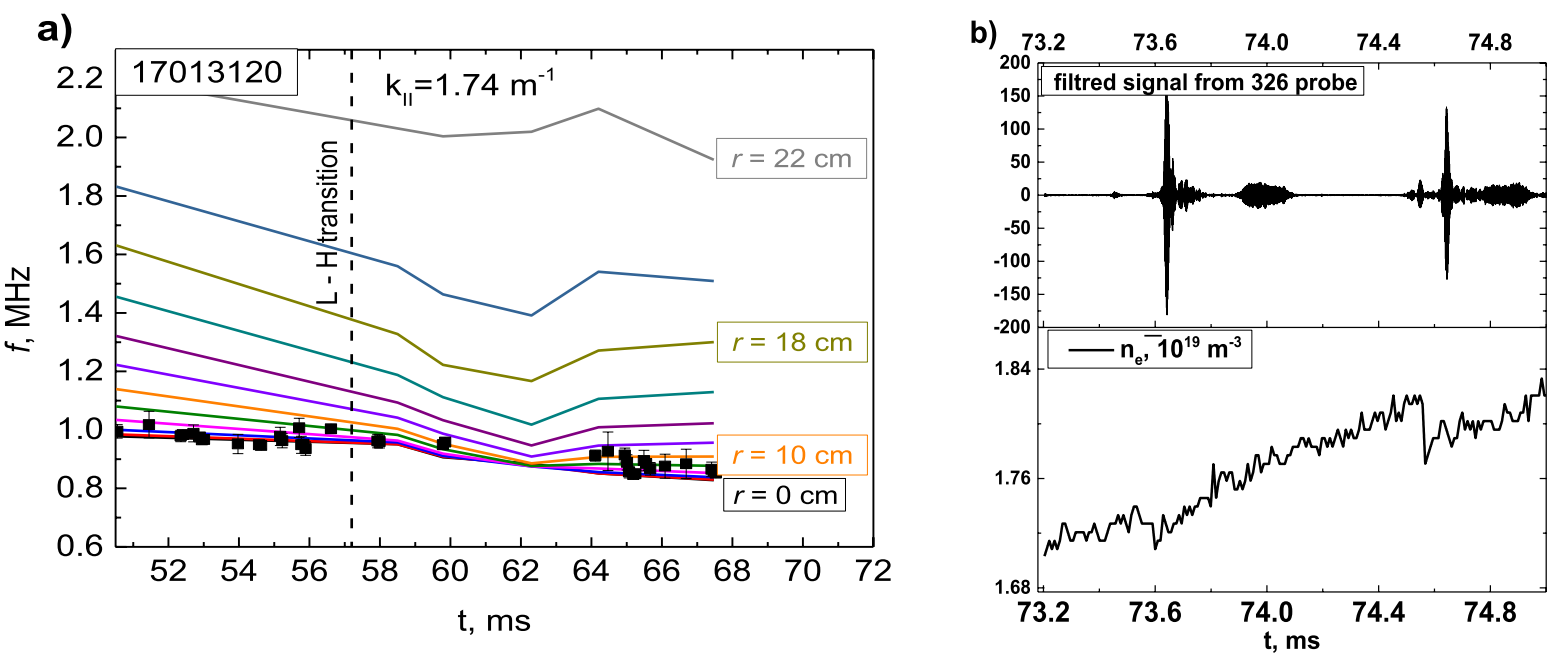

Figure 8. (a) Evolution of measured AW frequency (dots) and one calculated from local density evolution at different minor radii (lines) in a discharge with $\mathrm{L}-\mathrm{H}$ transition at $t=57.2 \mathrm{~ms},(b)$ correlation between sawtooth oscillations and AW bursts.
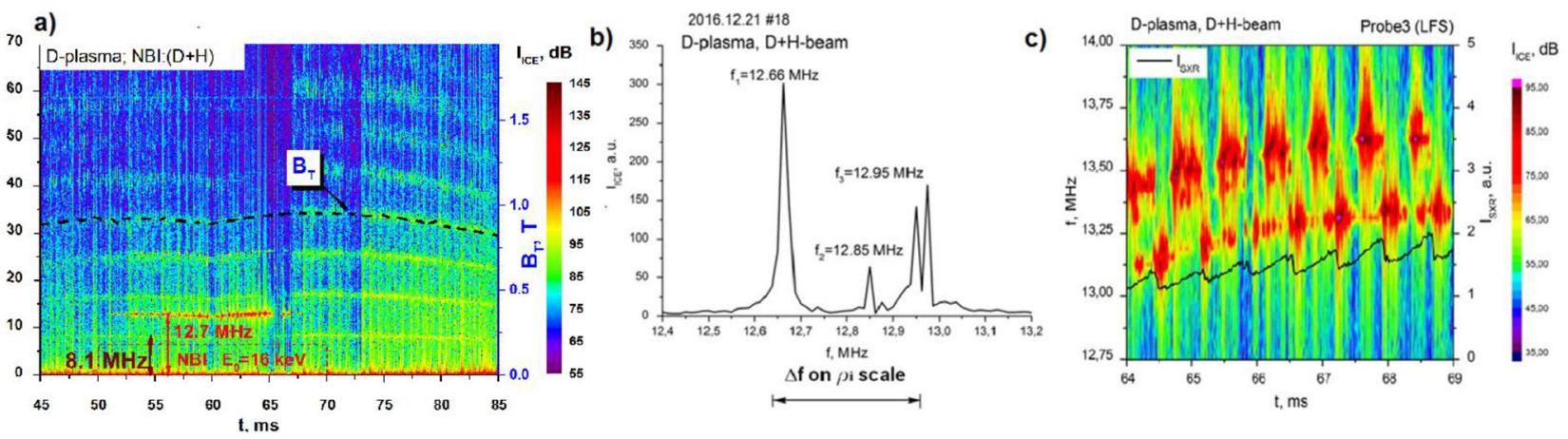

Figure 9. (a) Spectrogram of ICE in the TUMAN-3M deuterium plasma. NBI hydrogen ICE: thin bright line at $~ 12.5 \mathrm{MHz}$, with second and third harmonics barely seen. Ohmic deuterium ICE: broad line at $\sim 8.5 \mathrm{MHz}$ and its harmonics (up to seventh). (b) Fine structure of NBI ICE spectrum, $(c)$ evolution of spectral components of ICE caused by sawtooth activity.

These two types have a close frequency and similar poloidal spectrum, but different poloidal localization: short burst oscillations are localized predominantly at the top of the torus, whereas long bursts have a maximum amplitude at the bottom [31]. The TUMAN-3M AWs are driven not by NBI-produced fast ions (whose velocity is well below Alfvén velocity) but rather by magnetic field perturbation created by sawtooth crashes [32] or by loss of runaway electrons [33] associated with these crashes. Driving mechanisms for the two types of AWs may be different and require further investigation.

\section{Ion cyclotron emission}

Emission in the ion-cyclotron frequency range (ICE) in magnetically confined plasma is usually observed in the experiments where ion velocity distribution function is locally non-monotonic. Non-monotonic distribution is typically caused by NBI or radio frequency heating. In the Ohmic regime, ICE is observed in plasmas with an essential fraction of suprathermal ions that originated due to nuclear fusion reactions [34].

\subsection{ICE in NBI-heated plasma}

ICE, observed on the TUMAN-3M tokamak in the NBIheated scenario, is produced mainly by a minority of fast ions (e.g. fast deuterons in hydrogen plasma, and by fast protons in deuterium plasma) $[35,36]$. In contrast to other experiments, where ICE was found to originate mostly from the low field side (LFS) periphery, ICE in TUMAN-3M comes from the core plasma region. A rare example of core ICE can be seen in the recent ASDEX Upgrade experiments [37]. ICE frequency in TUMAN-3M was found to follow toroidal magnetic field evolution and to be insensitive to changes in plasma density. An experimentally observed ICE spectral line consists of two to four unevenly spaced very narrow lines, see figure 9 .

Most probably, ICE in TUMAN-3M is a result of compressional Alfvén eigenmode instability (with the dispersion relation $\omega=k v_{\mathrm{A}}$ ) excited by stagnation fast ions with narrow drift orbits localized in the vicinity of $R \sim R_{0}$ and having an average vertical drift velocity close to zero [38]. For these ions, having a velocity $v_{\mathrm{b}}$ and ion-cyclotron resonance frequency $\omega_{\mathrm{ci}}$, the Doppler-shifted resonance condition is $\omega=l \omega_{\mathrm{ci}}+k_{\|} v_{\mathrm{b}}$ [39]. Here $\omega$ and $k_{\|}$are the wave 


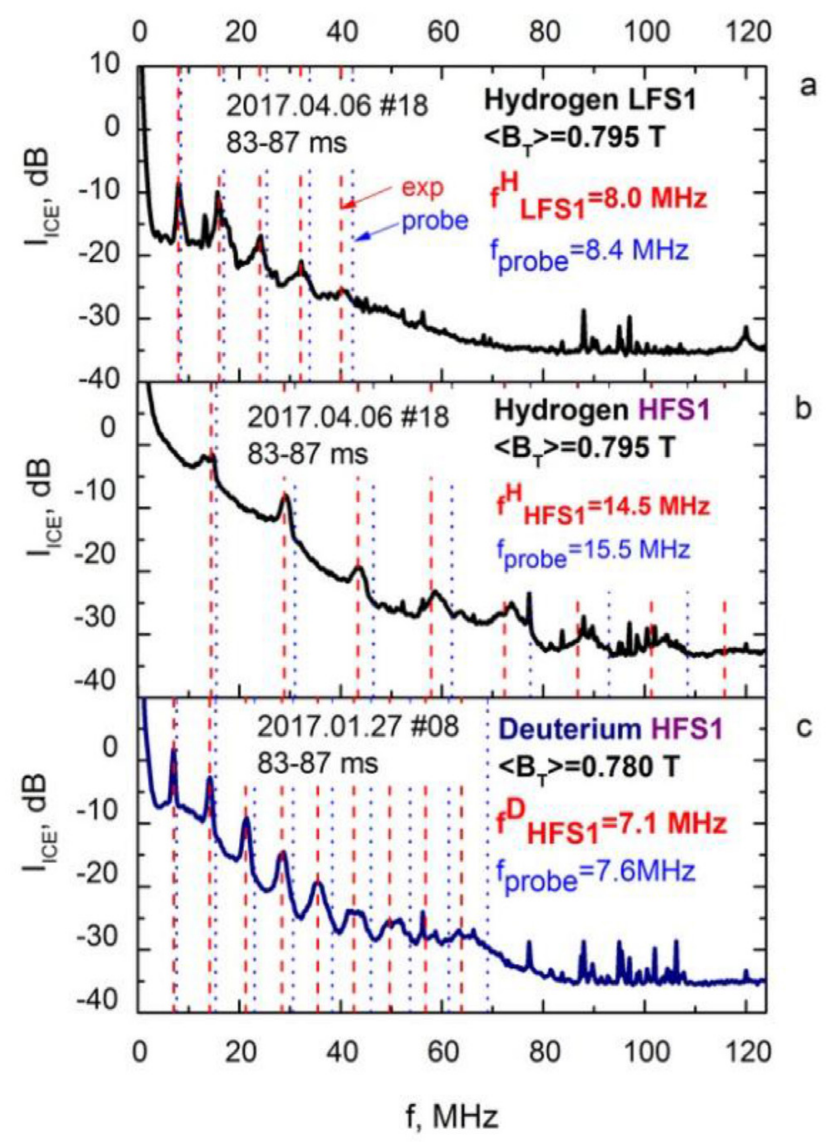

Figure 10. Spectra of OICE on the two magnetic probes: LFS1 and HFS1 (high field side) in D and H plasmas. Dotted lines denote calculated ion-cyclotron frequency $f_{\mathrm{ci}}$ and its harmonics.

frequency and parallel wave vector. The observed frequency of hydrogen ICE in deuterium plasma is $f \approx 13 \mathrm{MHz}$. This is slightly lower than the ICR frequency of fast ions, $\left(\omega-\omega_{\mathrm{ci}}\right) / 2 \pi=k_{\|} v_{\mathrm{b}} / 2 \pi \approx-1.5 \mathrm{MHz}$, meaning the wave is counter-propagating and has the toroidal mode number $n=-1$. Neutral beam, used on TUMAN-3M, has a nonmonoenergetic spectrum, comprising $E_{0}, E_{0} / 2, E_{0} / 3,2 E_{0} / 3$ and some other components. Fast ions, produced by ionization of these components, have different parallel velocities $v_{\mathrm{b}}$ and locate at slightly different stagnation orbits, resulting in different ICR and ICE frequencies. In this framework, the next experimentally observed characteristic features of TUMAN-3M NBI ICE can be understood: exact harmonics for $l=1,2,3 \ldots$; linear dependence of ICE frequency on toroidal field and virtually absent dependence on plasma density; and fine structure of the ICE frequency line.

\subsection{ICE in $\mathrm{OH}$ plasma}

Oscillations in the ion-cyclotron frequency range (5-100 MHz) were recently detected by fast magnetic probes in $\mathrm{OH}$ plasma in the TUMAN-3M tokamak. This phenomenon was called Ohmic ICE (OICE). The distinguishing feature of OICE is that it was not caused by suprathermal fusion products as the ion temperature was too low $\left(T_{\mathrm{i}} \sim 150-300 \mathrm{eV}\right)$. The following characteristic features of OICE were found.

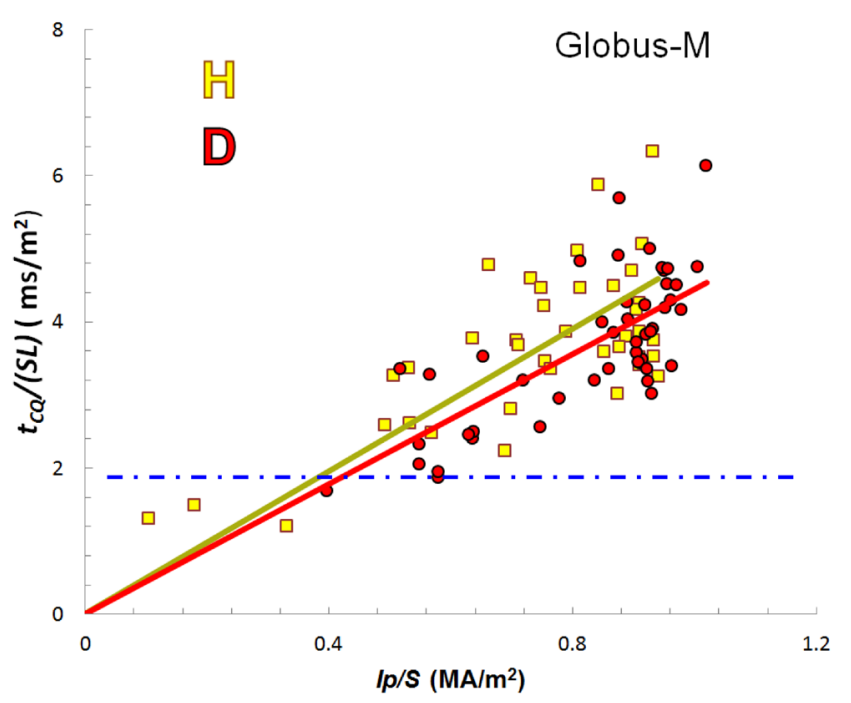

Figure 11. Current quench time normalized to $S$ and to the dimensionless plasma inductance $L$ versus $I_{\mathrm{p}} / S$ for hydrogen (squares) and deuterium (circles) plasmas. The horizontal dashdotted line shows the ITER critical value.

- The OICE was detected in the hydrogen and deuterium plasmas by magnetic probes, located inside the vacuum vessel, both in the inner (high magnetic field side) and outer (low field side) parts of the tokamak.

- The spectrum of the OICE typically consisted of many harmonics with frequencies evolving in time as the toroidal magnetic field $B_{\mathrm{T}}(t)$ (see the spectrogram in figure 9, where the harmonics of OICE are shown together with $\left.B_{\mathrm{T}}(t)\right)$.

- OICE intensity was distributed asymmetrically relative to the equatorial plane: it was not possible to register OICE below the equator.

- The fundamental frequency of OICE, registered by each of the probes, depends on the probe position; it corresponds to the ICR frequency of the main plasma ions in the close vicinity of the corresponding magnetic probe, regardless of whether hydrogen $\left(\mathrm{H}^{+}\right)$or deuterium $\left(\mathrm{D}^{+}\right)$ was the main ion (figure 10).

- NBI does not influence the fundamental frequency of OICE regardless of which hydrogen isotope atom is injected [35]; the injection does influence the intensity and harmonic content of OICE though.

In [40], the possibility of excitation of drift instabilities in inhomogeneous plasma at harmonics of the ion cyclotron frequency is shown. The model proposed in this work seems to be the most suitable for describing the phenomenon of OICE observed on the TUMAN-3M tokamak.

\section{Discharge disruption}

The characterization of plasma current quench during major disruption was studied in the Globus-M spherical tokamak. In contrast with the International Disruption Database for conventional tokamaks [41], experiments demonstrate a favorable, almost linear, dependence of the normalized current 


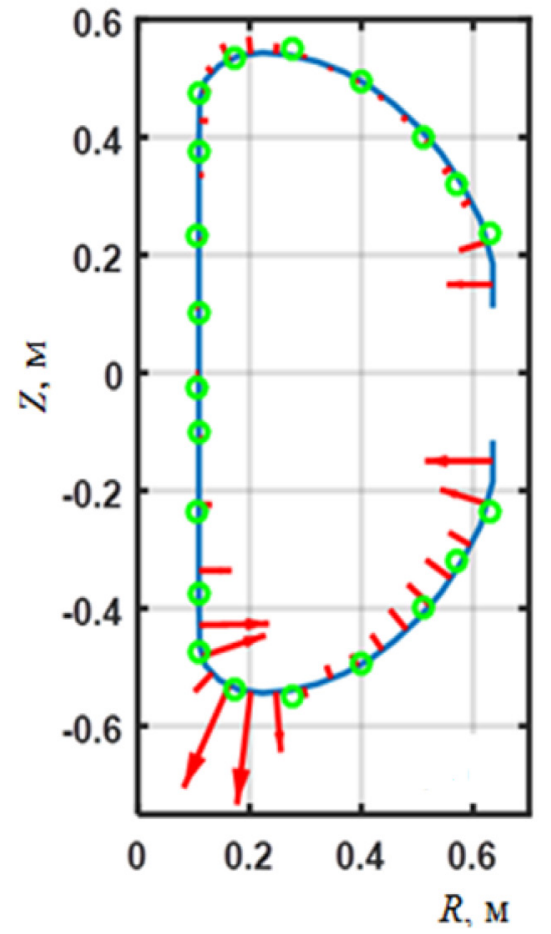

Figure 12. Distribution of the normal pressure on the Globus-M vessel. Circles show magnetic loops. Arrows are proportional to the pressure magnitude.

quench time $t_{\mathrm{CQ}} / S$ on the plasma current density $I_{\mathrm{p}} / S$ before the disruption [42], where $S$ is the area of the plasma poloidal cross-section. The minimum normalized disruption time in Globus-M, obtained at a very low plasma current, is shorter by a factor of 2.2 than the critical value adopted for ITER $t_{\mathrm{CQ}} / S=1.67 \mathrm{~ms} \mathrm{~m}^{-2}$. At a higher Globus-M plasma current the values of $t_{\mathrm{CQ}} / \mathrm{S}$ exceed ITER critical value several times. The additional division of $t_{\mathrm{CQ}} / S$ by the dimensionless inductance $L=\left[\ln \left(8 R / a \kappa^{1 / 2}\right)-1.75\right]$ ( $L \sim 1$ for conventional tokamaks, $L \sim 0.5$ for Globus-M), where $\kappa$ is the vertical elongation of the plasma column before the disruption, brings the Globus-M data closer to the data obtained for conventional tokamaks (figure 11). One can see from the figure that the normalized current quench time depends weakly on the ion mass.

The current quench is usually accompanied by the fast vertical displacement of the plasma column and results in a considerable toroidal eddy current in the vessel wall [43]. Interaction of the current with the poloidal magnetic field produces electromagnetic loads. To calculate the distribution of the eddy toroidal currents and electromagnetic pressures, a 2D mathematical model of the vessel wall was developed. In the model the wall is approximated by 64 axisymmetric rings with a certain electric resistance closed along the torus. The voltages, induced during the plasma current quench, were measured with a set of 21 loops, located on the vessel wall. Each loop was associated with a group of the neighboring wall elements. The distribution of the pressure, normal to the vessel wall, in the shot with downward plasma displacement during the disruption is shown in figure 12 . In the example the plasma current before the disruption is $I_{\mathrm{p}} \approx 0.2 \mathrm{MA}$ and the current quench time $\sim 0.6 \mathrm{~ms}$. The maximum local pressure
Table 1. Experimental $\lambda_{q}$ and scaling estimations.

\begin{tabular}{lllll}
\hline$B_{\mathrm{T}}, \mathrm{T}$ & $I_{\mathrm{p}}, \mathrm{kA}$ & $\lambda_{q}, \mathrm{~mm}$ & $\lambda_{q}^{\text {Eich-2011 }}, \mathrm{mm}$ & $\lambda_{q}^{\text {Eich-2011 }}, \mathrm{mm}$ \\
\hline 0.4 & 180 & $4.9 \pm 0.5$ & 7.0 & 4.0 \\
0.5 & 225 & $4.1 \pm 0.5$ & 5.4 & 3.4 \\
\hline
\end{tabular}

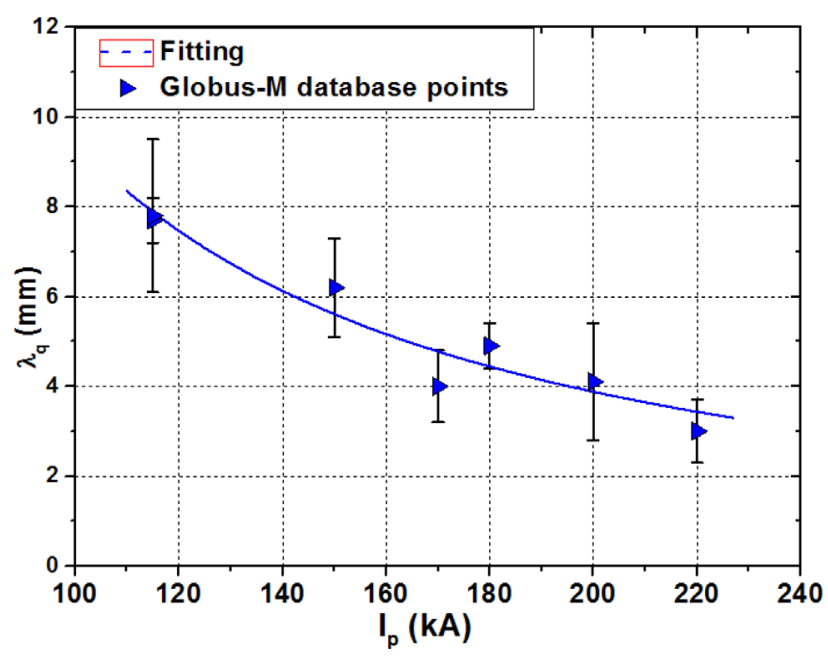

Figure 13. Globus-M fitting for power decay length via plasma current.

on the lower dome of the vessel is about $17 \mathrm{kPa}$, which is much lower than the atmospheric pressure. The pressures are directed oppositely over a short vessel section. Such a complicated distribution of the electromagnetic loads is connected with a strong inhomogeneity of the poloidal magnetic field in this region. The vacuum vessel is constructed using the membrane theory of shells, i.e. twisting and bending moments in the domes caused by the atmospheric pressure are small. However, during the current quench, a bending moment arises.

\section{SOL study}

A new nine-pin Langmuir probe, which can be moved along the major radius between shots, was installed at the outer midplane of the Globus-M tokamak. The probe head makes it possible to measure the following parameters: the floating potential $\left(V_{\mathrm{f}}\right)$, ion saturation current $\left(I_{\mathrm{is}}\right)$, electron temperature $\left(T_{\mathrm{e}}\right)$, electron density $\left(n_{\mathrm{e}}\right)$ and the Mach number $\left(M_{\|}\right)$. A detailed description of the probe can be found in [44]. One of the main advantages of this probe is its resistance to high thermal loads.

One of the crucial parameters of the edge SOL plasma, determining the heat loads to the divertor plates, is a power decay length $\lambda_{q}$. A full theoretical model, allowing evaluation of this parameter, does not exist yet and empirical scalings are usually used for this purpose [45]. According to a simple SOL approach [46] the power flux is proportional to $n_{\mathrm{e}} T_{\mathrm{e}}^{3 / 2}$. Therefore, power decay length at the midplane could be calculated using the following expression: $\lambda_{q}=$ $\left(1 / \lambda_{\mathrm{ne}}+\left(3 /\left(2 \lambda_{\mathrm{Te}}\right)\right)^{-1}\right.$, where $\lambda_{\mathrm{ne}}$ and $\lambda_{\mathrm{Te}}$ are electron density and temperature scale lengths respectively. Electron temperature and density profiles in SOL were measured for the two 


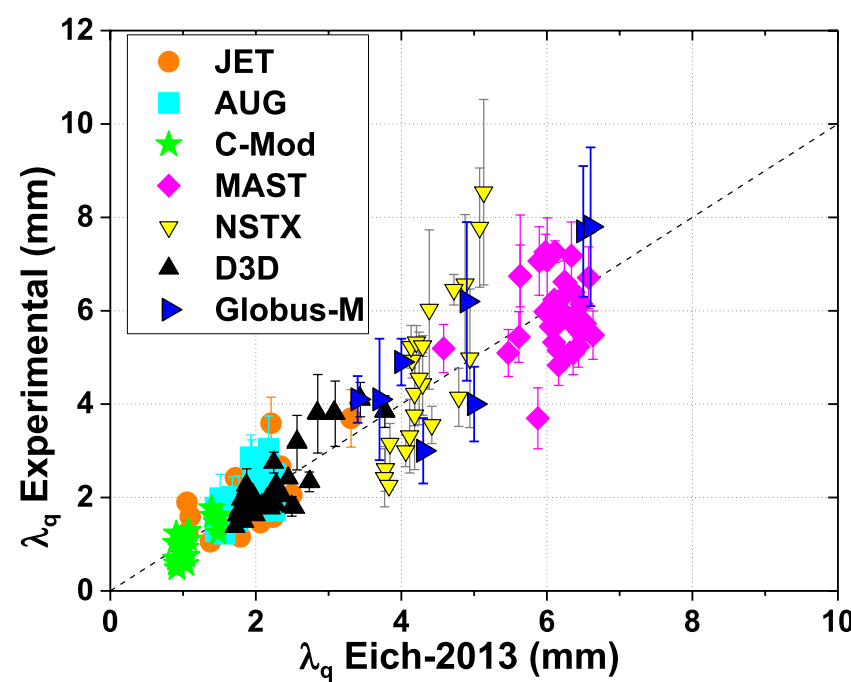

Figure 14. Comparison of the estimated $\lambda_{q}$ in Globus-M and other tokamaks with predictions by Eich-2013 scaling.

series $\left(B_{\mathrm{T}}=0.4 \mathrm{~T}, I_{\mathrm{p}}=180 \mathrm{kA}\right.$ and $B_{\mathrm{T}}=0.5 \mathrm{~T}, I_{\mathrm{p}}=225$ $\mathrm{kA}$ ) of the lower single-null discharges with close $q_{95}$ values. The results were compared with Eich's scalings of 2011 [45] and 2013 [47] (see table 1). Like in the previous experiments [48], derived values of $\lambda_{q}$ are closer to the Eich-2013 scaling. The dependence of $\lambda_{q}$ on plasma current was updated using the Globus-M database with plasma current values from 115-250 kA: $\lambda_{q} \sim I_{\mathrm{p}}^{-(1.3 \pm 0.2)}$ (figure 13) [22]. In figure 14, estimated $\lambda_{q}$ in Globus-M is compared to $\lambda_{q}$ in other tokamaks. It can be seen that power decay length dependence on $I_{\mathrm{p}}$ in Globus-M is consistent with the Eich-2013 scaling predictions.

\section{Benchmarking of full-f global gyrokinetic model- ing results against the FT-2 tokamak DR data using synthetic diagnostics}

The massively parallelized particle-in-cell simulations of the gyrokinetic (GK) distribution function and the electric field provide an efficient theoretical tool for studying the nonlinear turbulent plasma dynamics, which, however, need validation and comprehensive benchmarking against the experimental data. In this section the results of the global GK particle-in-cell simulations, performed by ELMFIRE code, are quantitatively compared with the X-mode DR experimental data obtained at the high magnetic field side of the FT-2 tokamak [49]. Two versions of the DR synthetic diagnostics, a fast linear version based on the reciprocity theorem [50] and a full-wave version utilizing the IPF-FD3D code [51] are used for this purpose. The experimental data obtained with standard DR, utilizing a variable antenna position, and with the radial correlation DR (RCDR), which was used in the comparison, characterize the tokamak plasma turbulent dynamics at different scales: micro(density fluctuations of trapped electron mode), macro- (the velocity of global plasma flows) and meso-scale (GAM).

The benchmarking against X-mode DR experimental data has demonstrated a good agreement between the DR spectra

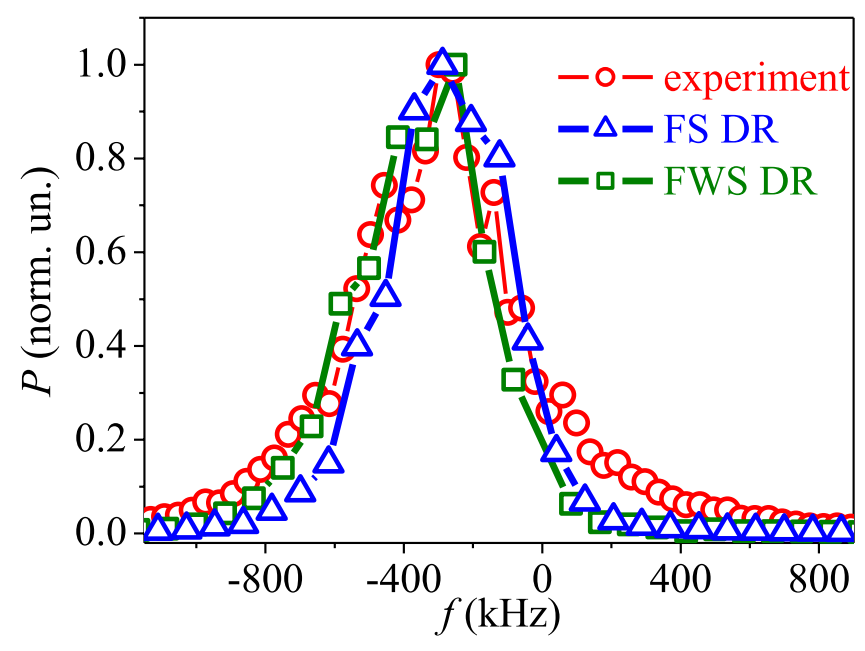

Figure 15. Comparison of DR spectra for up-shifted antenna vertical displacement. Circles-experiment; triangles-fast synthetic DR, squares—full-wave synthetic DR.

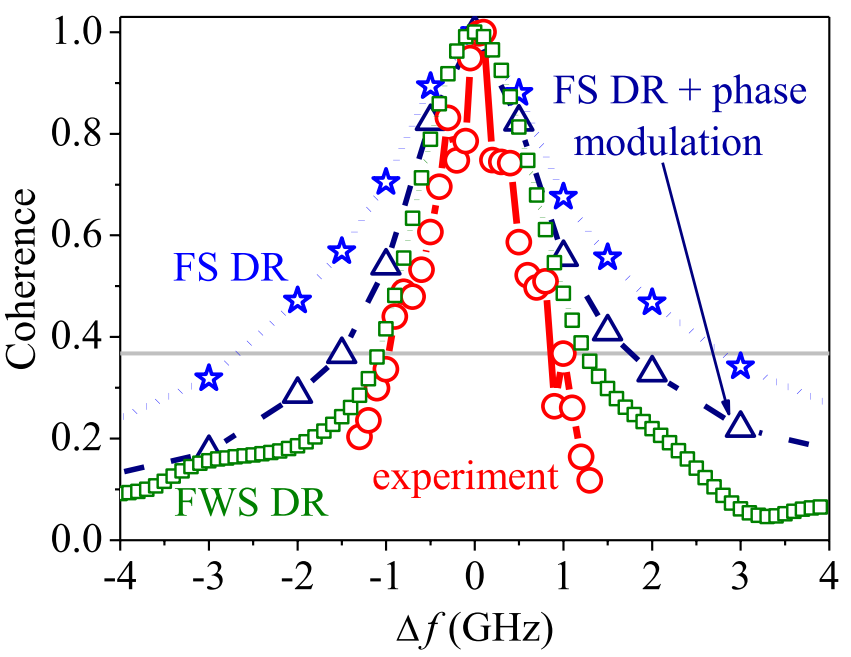

Figure 16. The RCDR CCF against the channel frequency separation. Stars-fast synthetic DR; squares-full-wave synthetic DR; triangles - fast synthetic DR with phase modulation; circlesexperiment.

measured and computed using both synthetic diagnostics (see figure 15). For all antennae positions used for comparison, both the spectra frequency shift and width, and in many cases the spectra shape, were similar, thus demonstrating a correct reproduction of the electric field behavior in the FT-2 tokamak by the ELMFIRE GK code. The mean fluctuation velocities, determined by the DR measurements and the synthetic diagnostics, were close. The GAM frequency and amplitude, provided by the measurements and by the synthetic DR, appear to be close within a $12 \%$ accuracy. However, a drastic (factor of three) excess was found in the decay with a growing frequency shift in the probing wave channels of the RCDR crosscorrelation function $(\mathrm{CCF})$ provided by the fast synthetic diagnostics compared to the experimental one. The quick decrease of the radial correlation observed in the experiment is attributed to the phase modulation of the probing wave due to the long-scale density fluctuations, which is shown to be close to $\pi / 3$ by both the specially performed measurements 


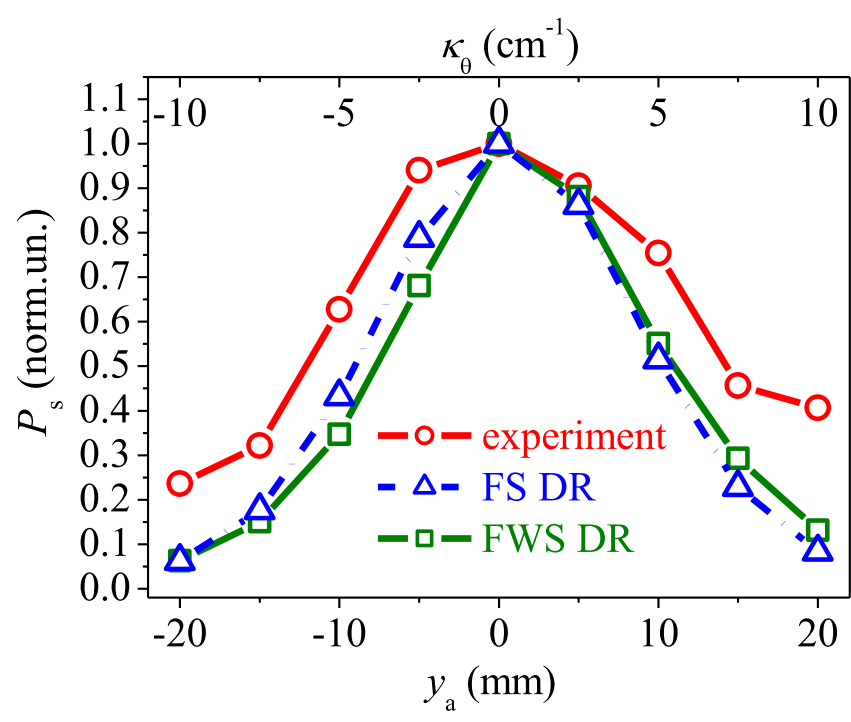

Figure 17. Dependence of the backscattering power on the fluctuation poloidal wavenumber or vertical antenna displacement $y_{\mathrm{a}}$.

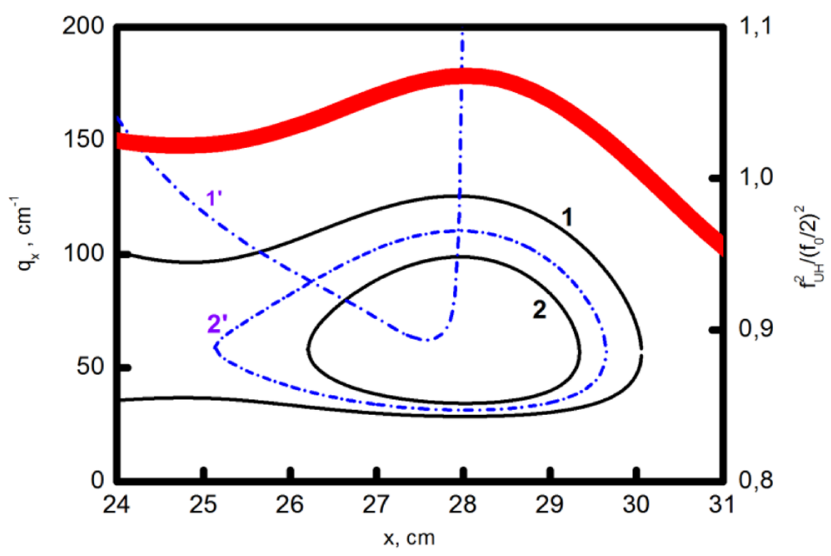

Figure 18. The dispersion curves of primary and secondary daughter waves generated in the cascade of decays; 1 - primary non-trapped UH wave, 2-primary trapped UH wave, $1^{\prime}$ - secondary IB wave, $2^{\prime}$ - secondary trapped UH waves. The dimensionless UH frequency profile $\left(f_{\mathrm{UH}}^{2} /\left(f_{0} / 2\right)^{2}\right)$ - thick solid curve.

and the Wentzel-Kramers-Brillouin estimation based on the GK modeling results. In spite of the fact that this value indicates only the beginning of the transition to the fluctuation reflectometry nonlinear regime $[52,53]$ (which therefore is not influencing the DR spectra) it already has a strong impact on the RCDR performance. This conclusion is confirmed by the full-wave DR synthetic diagnostics correctly accounting for the perturbation to the probing wave propagation produced by the turbulence, which demonstrates a much better agreement with the experimental CCF, as shown in figure 16. The only pronounced difference between the measurements and synthetic diagnostics is found in the dependence of the DR signal power on the antenna vertical displacement, which is related to the density fluctuation poloidal wavenumber spectrum (see figure 17). Dependences of the measured and synthetic DR signal power on the antenna vertical displacement are well approximated by Gaussian curves. However, the estimated 'spectral width', corresponding to these dependences,

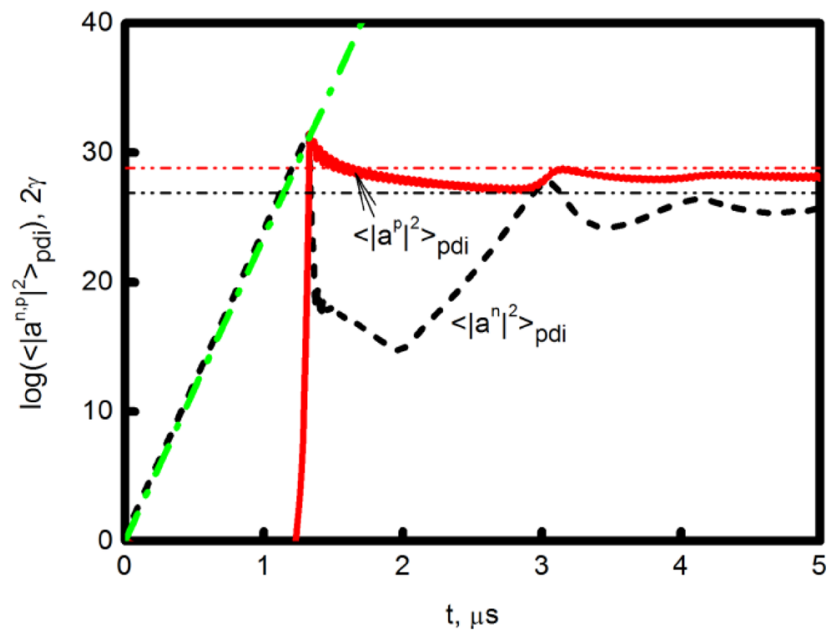

Figure 19. Temporal evolution of the averaged energy density of primary (dashed curve) and secondary (solid curve) UH plasmons. The dash-dotted and dash-double-dotted lines are the analytical predictions for the instability exponential growth and saturation stages, respectively.

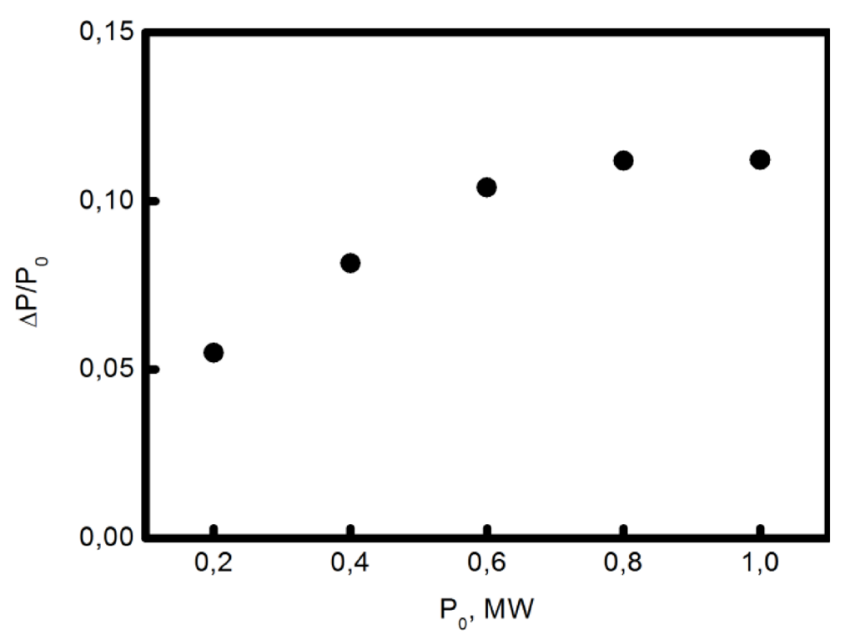

Figure 20. The anomalous absorption rate versus the pump power.

is different by a factor of 1.5 . This difference could be attributed to the fact that GK computations underestimate the smallscale turbulence level in its decay region at the high field side of the torus.

\section{Anomalous absorption and emission in ECRH experiments due to parametric excitation of localized UH waves}

In contrast to the standard theory [54] predicting high thresholds of anomalous phenomena at ECRH in tokamaks, anomalous backscattering was observed a decade ago at TEXTOR in modest power level X2-mode neo-classical magnetic island control experiments [55]. This effect was explained in [56] by the low-threshold parametric excitation of two UH waves trapped in the vicinity of a local maximum of plasma density situated in the magnetic island. However, it should be mentioned that the anomalous backscattering effect was observed at TEXTOR in a wide range of the plasma densities in the 


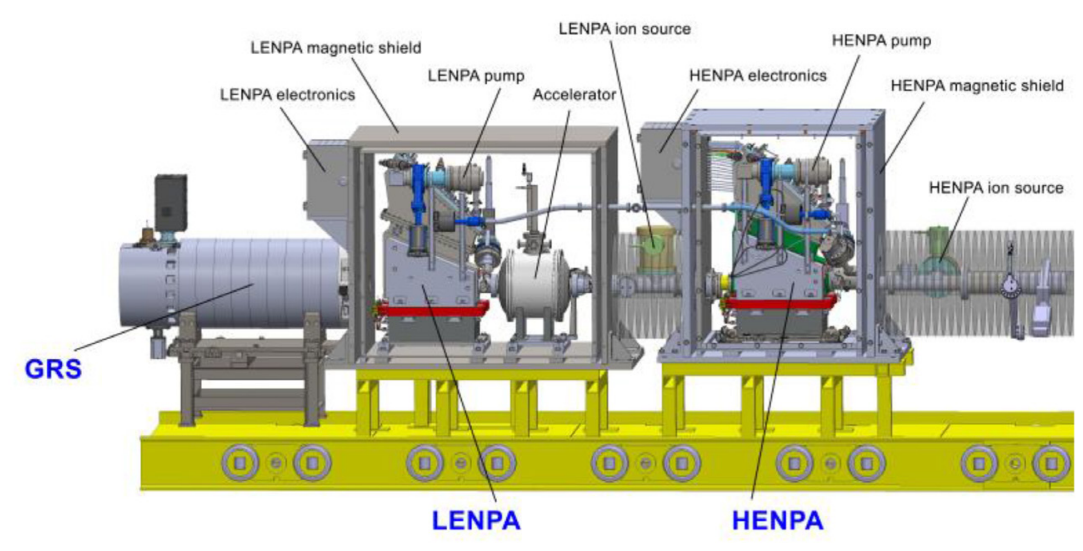

Figure 21. 3D design of the ITER NPA diagnostic system. LENPA and HENPA neutral particle analyzers and the GRS are shown.

local maximum of the density profile substantially exceeding the UH value for half the pump wave frequency. Under these more general conditions the trapping of both decay UH plasmons is no longer possible. Nevertheless, one of the primary UH daughter waves can still be localized, which can lead to the excitation of low-threshold absolute parametric two-plasmon decay instability (TPDI) [57]. In this paper we analyze the saturation of the TPDI under the general conditions when only one of the parametrically driven plasmons is trapped, whereas the second one can leave the decay region (see figure 18). We consider the secondary decay instability of the localized UH wave producing another $\mathrm{UH}$ wave and an ion Bernstein wave (see figure 19) as a moderator of the primary TPDI. The general analytical consideration is accompanied by numerical analysis performed under the experimental conditions typical of the off-axis X2-mode ECRH experiments at TEXTOR [55] demonstrating the instability saturation (see figure 19). We also estimate a fraction of the pump power $P_{0}$ gained anomalously throughout TPDI in this case. As shown in figure 20, well above the TPDI threshold, equal for the TEXTOR parameters to $128 \mathrm{~kW}$, the anomalous absorption rate is equal to $11 \%$.

It should be also underlined that in the case $\omega_{0} \simeq$ $2 \omega_{\mathrm{UH}}\left(x_{\mathrm{min}}\right)$ considered in this paper the non-trapped $\mathrm{UH}$ wave propagating outward perpendicular to the magnetic field is converted into the X-mode (see figure 18). It crosses the ECR surface and leaves the plasma at the high-field side. Neglecting the ECR absorption of the X-mode, which is small at the perpendicular propagation in the modest temperature plasma of middle scale devices, and taking into account the power balance in the decay by a factor $\alpha$ we can estimate the power of this $\mathrm{X}$-mode radiation at the half-pump frequency as $P_{\omega_{0} / 2}^{X}=\alpha P_{0} / 2$. In the case considered in this paper it results in $0.05 P_{0}$. The larger part of this power is reflected from the device wall in the form of the X-mode and finally absorbed after conversion in the UH resonance. However, a smaller part of the power, characterized by the cross-polarization factor $\beta_{\mathrm{XO}}$, is reflected in the form of an O-mode, which is partly absorbed in the ECR and then leaves the plasma. Taking into account the $\mathrm{O}$-mode absorption rate $\Gamma_{\mathrm{O} 1}$ we obtain the estimation for the $\mathrm{O}$-mode emission power at the low field side at the half pump frequency $Q_{\mathrm{O}} \approx 0.5 \alpha \beta_{\mathrm{XO}} \exp \left(-\Gamma_{\mathrm{O} 1}\right) P_{0}$.
Considering that for the conditions of the TEXTOR off-axis ECRH experiment $\Gamma_{\mathrm{O} 1}=2$, and assuming $\beta_{\mathrm{XO}}=0.01$, we get for the case under consideration $Q_{\mathrm{O}} \approx 8 \times 10^{-5} P_{0}$. Thus at the maximal microwave power of $600 \mathrm{~kW}$ one can expect to see the O-mode emission of $50 \mathrm{~W}$ at the half pump frequency at the low field side of the tokamak. This emission is observable only in the narrow density range when the UH density for half the pump frequency is slightly lower than plasma density in the profile local minimum, however the decay with the upper branch of the non-localized UH wave (electron Bernstein wave) is still possible. Out of this density range the intensive emission of sub-harmonic $\omega_{0} / 2$ is not possible; nevertheless due to the nonlinear coupling of UH plasmons and the pump wave emission of the $3 \omega_{0} / 2$ harmonic it can occur. It happens when the following conditions hold: $\omega_{\mathrm{ce}}\left(x_{\mathrm{m}}\right) \sqrt{\frac{n_{\mathrm{e}}\left(x_{\max }\right)}{\delta n_{\mathrm{e}}}}<\frac{5 \omega_{0}}{2}<\frac{c}{l_{\mathrm{T}}} \sqrt{\frac{\delta n_{\mathrm{e}}}{n_{\mathrm{e}}\left(x_{\max }\right)}}$, where $\delta n_{\mathrm{e}}=n_{\mathrm{e}}\left(x_{\max }\right)-n_{\mathrm{e}}\left(x_{\min }\right), n_{\mathrm{e}}\left(x_{\max }\right)$ and $n_{\mathrm{e}}\left(x_{\min }\right)$ stand for the density value in the local maximum and minimum of the profile, respectively. We get the $3 \omega_{0} / 2$ pump harmonic emission power received by the antenna as $p_{\mathrm{s}}=T_{3 / 2} P_{0}$, where the conversion coefficient at the TEXTOR parameters is equal to $T_{3 / 2}=5 \times 10^{-5}$. The maximal power utilized in the TEXTOR magnetic island control experiment results in $30 \mathrm{~W}$ of received $3 \omega_{0} / 2$ pump harmonic emission. More details on the research described in this paragraph can be found in [58].

\section{ITER diagnostics development}

Three diagnostics for ITER are being developed at the Ioffe Institute: neutral particle analysis (NPA), gamma-ray spectrometry and DTS combined with LIF.

\subsection{NPA and GRS}

Over the past few years, considerable progress has been made in several areas of NPA diagnostic system development. The current 3D design of the NPA system, which consists of lowenergy (LENPA) and high-energy (HENPA) neutral particle analyzers and a gamma-ray spectrometer (GRS), is presented in figure 21. A detailed technological analysis of the NPA design was done to conform with French nuclear regulations 


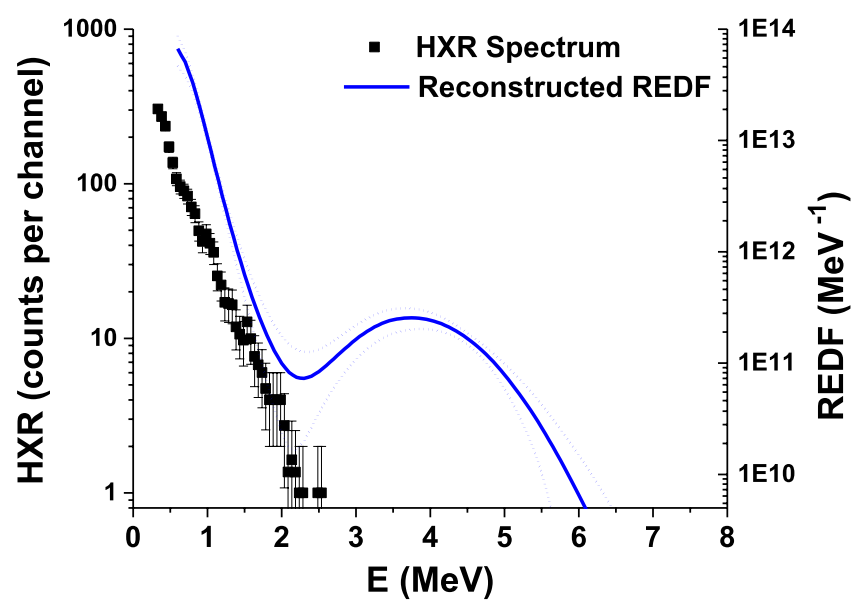

Figure 22. HXR spectrum recorded during an Ohmic TUMAN-3M discharge (squares) and the runaway electron distribution function (solid line) with uncertainties (dotted lines) calculated with the DaGaSum code.

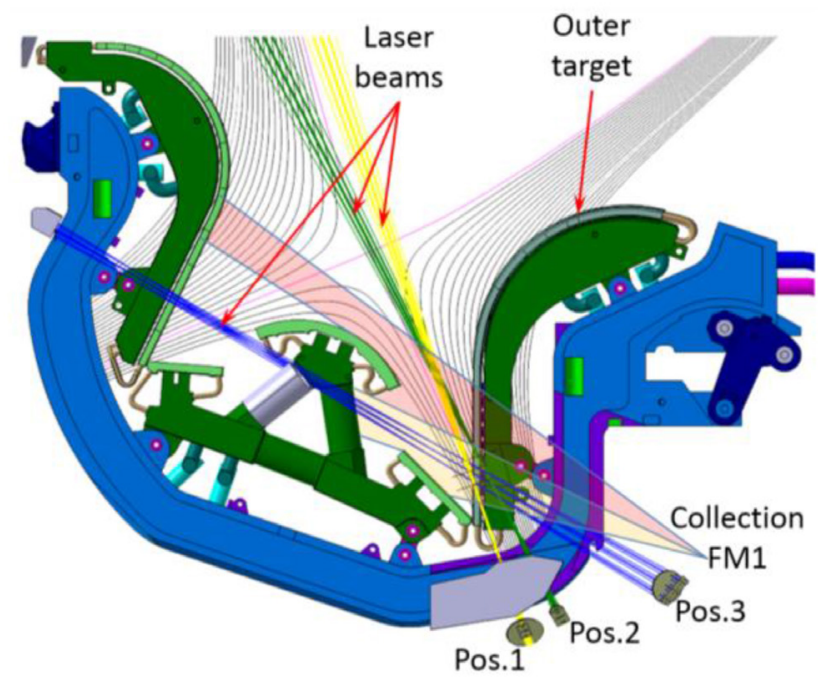

Figure 23. Optical layout of DTS/LIF probing chords in the ITER divertor. Pos. 1, 2 and 3 mark the first laser mirrors launching laser beams in outer leg plasma. Collection FM1 is the centrum of the collection aperture diaphragm.

and from the point of view of manufacturing. A lot of effort has been made in integrating LENPA and HENPA analyzers with their supporting subsystems into the ITER environment. At present, the tests of the most critical NPA components are underway [59], and the problems related to NPA neutron and magnetic shield design are being resolved [60]. One of the main goals for the analyzers at ITER is the measurement of the fuel isotope $(D / T)$ ratio [61]. The influence of various plasma phenomena on the NPA ratio measurement has therefore been analyzed. It was shown that NBI [62], pellet injection [63] and sawtooth oscillations [64] may have some effect on NPA signals, which should be taken into account by appropriate modelling to get sufficiently accurate $\mathrm{D} / \mathrm{T}$ measurements.

A GRS is placed in the neutron dump of the NPA system behind LENPA (figure 21). The spectrometer is intended to diagnose accelerated ions and electrons by the line-integrated gamma-ray measurements [27, 65]. They will provide complementary signals for NPA data on fuel ratio and energy spectra of confined $\alpha$ - and other energetic particles (p, D, T, ${ }^{3} \mathrm{He}$ ). The GRS includes two detectors: a semiconductor high-purity Ge detector and a scintillation detector with a $\operatorname{LaBr}_{3}(\mathrm{Ce})$ crystal. For spectrometric measurements under the conditions of a thermonuclear experiment it was necessary to develop an algorithm for digital signal processing, providing stable measurements at the counting rate of $\operatorname{LaBr}_{3}(\mathrm{Ce})$ up to $5 \times 10^{6} \mathrm{~s}^{-1}$ and up to $5 \times 10^{5} \mathrm{~s}^{-1}$ for the HPGe detector. The developed data acquisition system based on the NI PXIe architecture makes it possible to record the detector signal for at least $400 \mathrm{~s}$ with a sampling rate of $250 \mathrm{MHz}$. At present, the prototyping and testing of the main components of the GRS are carried out under conditions close to the ITER ones. The $\operatorname{LaBr}_{3}(\mathrm{Ce})$ detector mockup was successfully tested for a radiation resistance under an integral neutron flux of $1.1 \times 10^{13} \mathrm{~cm}^{-2}$. More details on the GRS development are presented in [66].

To provide energy distributions of fast electrons and ions in tokamak plasmas based on gamma-ray measurements, a spectrum deconvolution code DeGaSum has been developed at the Ioffe Institute [67]. The code was applied to get the energy distributions of runaway electrons in experiments at FT-2 [68, 69], TUMAN-3M [70], JET [71] and ASDEX-Upgrade [72]. Examples of a hard X-ray spectrum recorded during an Ohmic TUMAN-3M discharge and the runaway electron distribution function reconstructed with the DeGaSum code are shown in figure 22. The gamma-ray spectra deconvolution algorithm, based on the measurement of intensities of peaks from different reactions, involving deuterium, was used for the reconstruction of the ion cyclotron resonance heating (ICRH)-accelerated $\mathrm{D}^{+}$distribution on JET [73].

\subsection{DTS combined with LIF}

The ITER DTS and LIF are active laser-aided diagnostics providing local measurements of plasma parameters in the outer divertor leg (see figure 23), i.e. in the area with extremely steep gradients, both parallel and perpendicular to the magnetic surfaces. The combined DTS/LIF diagnostic provides simultaneous measurement of the local $T_{\mathrm{e}}, n_{\mathrm{e}}, T_{\mathrm{i}}, n_{\mathrm{i}}, n_{\mathrm{a}}$ along the probing laser paths with a resolution of $25 \mathrm{~mm}$ along the probing chords.

SOLPS modeling of detachment in the ITER divertor requires a detailed knowledge of:

- electron processes, including rates of ionization, recombination and radiation;

- ion-neutral collisions responsible for: control of effective pressure in the recycling region, with counter-balancing of the upstream plasma pressure; cooling the plasma down to $\sim 1 \mathrm{eV}$ and initiating the recombination processes (without recombination each ion reaching the plates will transfer $13.6 \mathrm{eV}$ in the form of heat); and 'friction' switching the plasma flow from free streaming to diffusion, making the residence time of the electrons and ions sufficient for recombination. 

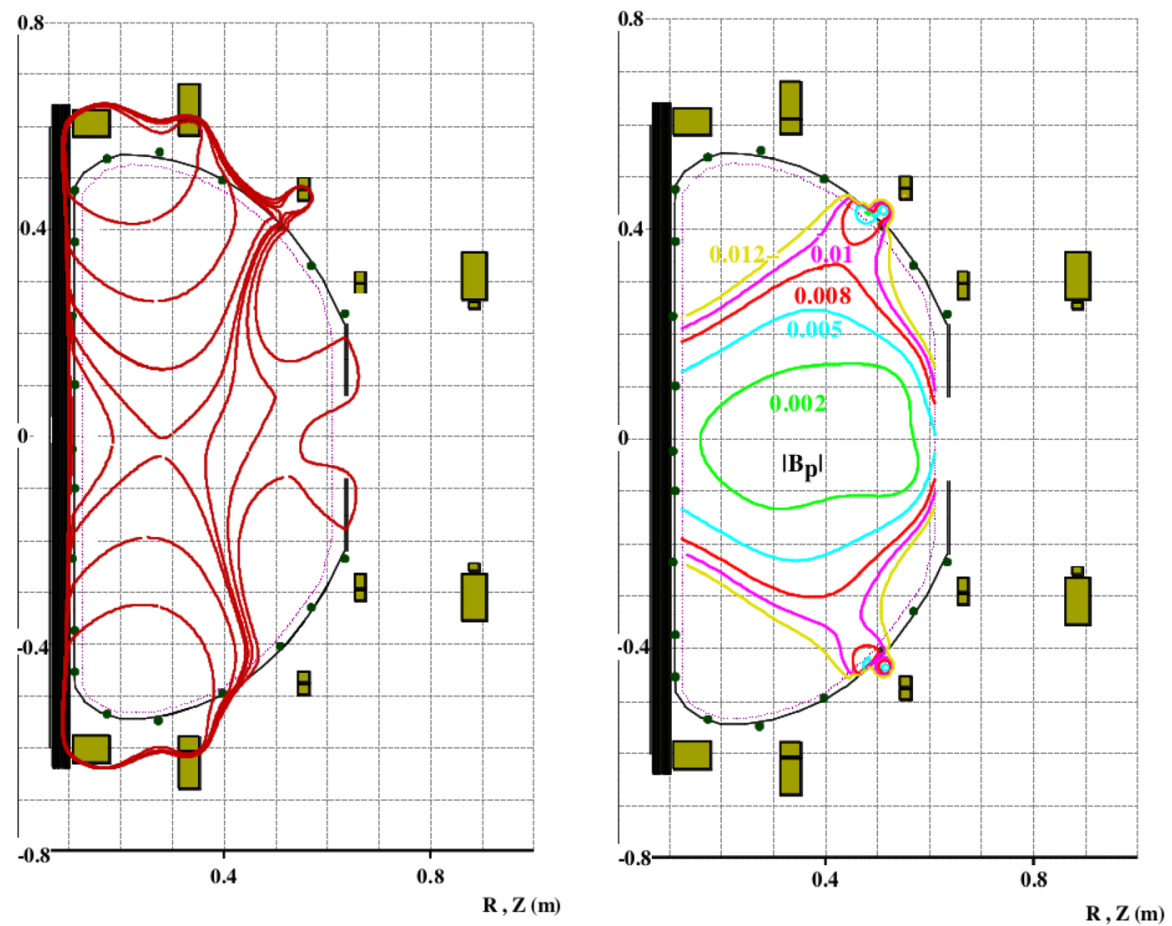

Figure 24. The reconstruction of the magnetic flux lines (left) and of the poloidal magnetic field $\left|B_{\mathrm{p}}\right|=0.002,0.005,0.008,0.010,0.012 \mathrm{~T}$ (right) at the moment of plasma breakdown.

Measuring $T_{\mathrm{e}}, n_{\mathrm{e}}, T_{\mathrm{i}}, n_{\mathrm{i}} n_{\mathrm{He} / \mathrm{H} / \mathrm{D} / \mathrm{T}}$ simultaneously, the following important parameters in the respective points of the divertor SOL can be calculated:

- ionization balance: rates of ionization and recombination $\left(T_{\mathrm{e}} n_{\mathrm{e}} n_{\mathrm{He} / \mathrm{H} / \mathrm{D} / \mathrm{T}}\right)$;

- emission intensity $\left(T_{\mathrm{e}} n_{\mathrm{e}} n_{\mathrm{i}} n_{\mathrm{He} / \mathrm{H} / \mathrm{D} / \mathrm{T}}\right)$;

- frictional forces determined by collisions with neutrals $\left(T_{\mathrm{i}} n_{\mathrm{i}} n_{\mathrm{He} / \mathrm{H} / \mathrm{D} / \mathrm{T}}\right)$;

- pressure of the incoming plasma flow $\left(T_{\mathrm{e}} n_{\mathrm{e}} T_{\mathrm{i}} n_{\mathrm{i}}\right)$.

Challenges of DTS implementation in the ITER divertor were discussed in [74] together with its capability to satisfy project requirements, related to the range of the measured $T_{\mathrm{e}}$ and $n_{\mathrm{e}}$. The main challenge for DTS is extremely high $n_{\mathrm{e}}$ and low $T_{\mathrm{e}}$ plasmas in the regions with predominant recombination. The developed approach, based on synthetic experiments, shows that the expected $T_{\mathrm{e}}$ and $n_{\mathrm{e}}$ measurement accuracy is better than the specified technical requirements, in spite of the pronounced collective effects [75]. Temporal shapes of the atomic helium fluorescence can be also utilized for the measurement of $n_{\mathrm{e}}$ in the range of $10^{18}-10^{20} \mathrm{~m}^{-3}$. The dynamic collision-radiative model, developed for helium, allows for fitting a calculated temporal shape to the measured fluorescence signals. It was verified in Globus-M experiments, showing a good agreement between TS and LIF for $T_{\mathrm{e}} \sim 110 \mathrm{eV} n_{\mathrm{e}} \sim 2 \times 10^{19} \mathrm{~m}^{-3}$ [75]. Laser-induced quenching is a new technique [75], proposed for the measurement of $\mathrm{H} / \mathrm{D} / \mathrm{T}$ atomic density in the ITER divertor. The current status of LIF performance of $T_{\mathrm{i}}$ and $n_{\mathrm{He}}$ measurements using HeI and HeII fluorescence was presented in [76]. There have been multiple recent achievements in the hardware development.
The progress in the development of DTS filter polychromators and piezomotors is presented in [77]. The most recent achievements in the development of DTS laser system were presented in [78]. Investigation of laser resistance of plasmafacing diagnostic components was discussed in [79].

\section{Globus-M2}

Globus-M2 is an upgraded version of the Globus-M tokamak with the same vacuum vessel and an enhanced electromagnetic system and power supplies. Auxiliary plasma heating, current drive systems and a set of diagnostics were upgraded as well. An increase in the magnetic field (from 0.5 to $1.0 \mathrm{~T}$ ) together with an increase in the plasma current (from 0.25 to $0.5 \mathrm{MA}$ ) in the Globus-M2 tokamak should improve plasma performance and provide enhanced conditions for auxiliary heating and current drive [48, 80-84]. ICRH in the case of an increased magnetic field (and, as a result, the growth in the resonant frequency up to $15 \mathrm{MHz}$ ) become more efficient due to the enhancement of the single-pass absorption and the improvement of the antenna-plasma coupling. An increase in the efficiency of an NBI is expected due to a significant reduction in fast particle losses. Approximately half of the plasma current can be sustained non-inductively by simultaneous injection of the two $1 \mathrm{MW}$ neutral beams. The use of the lower-hybrid waves $(2.45 \mathrm{GHz})$ with an input power level of 0.5 MW should ensure a completely non-inductive maintenance of current in the discharge. Eventually an improvement in the plasma parameters, including an increase in plasma temperature and significant drop in collision frequency, is expected. 


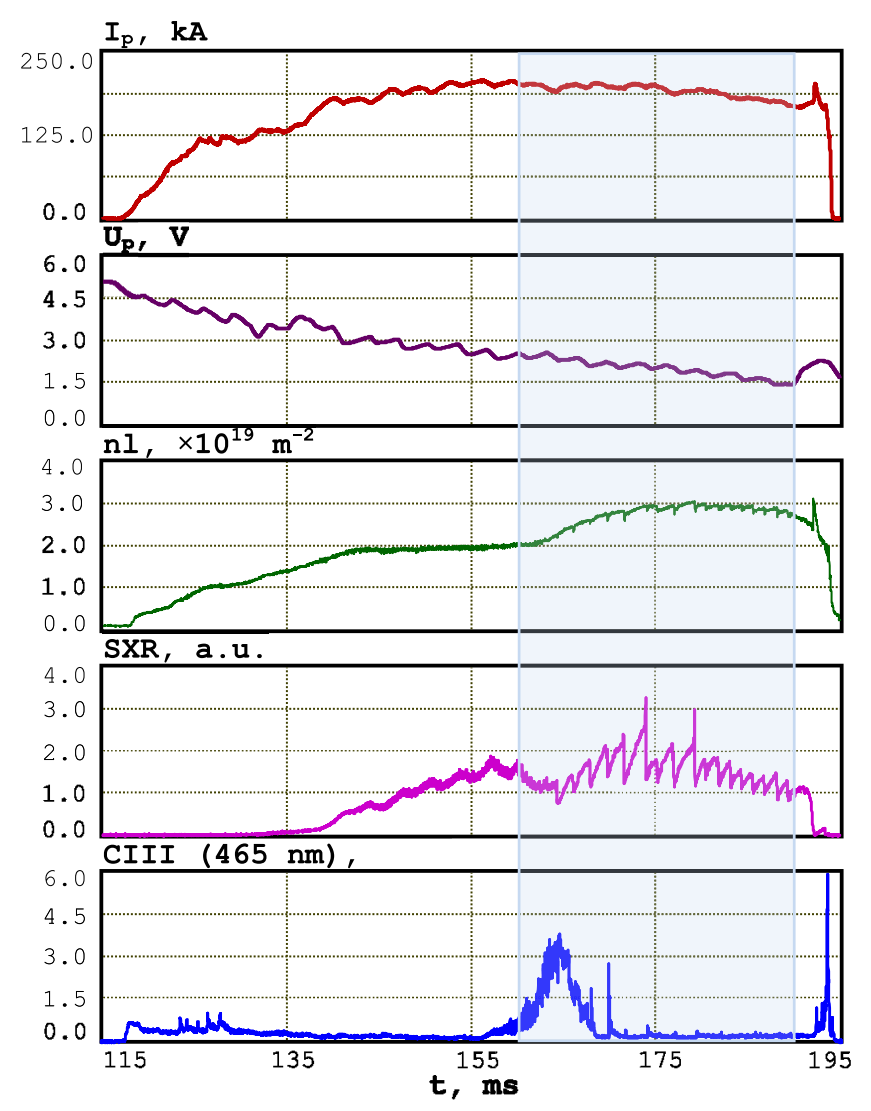

Figure 25. The evolution of the plasma current, loop voltage, line averaged density, soft $\mathrm{x}$-ray signal and CIII emission intensity in shot \#37269 with the $25 \mathrm{keV}$, 0.6 MW D-NBI (transparent filling).

In the frame of preparation for the first plasma experiment $[85,86]$, the breakdown was optimized. It was possible to provide a significant area with a 'zero' value (below $2 \mathrm{mT}$ ) of the magnetic field poloidal component in the tokamak discharge vessel (see figure 24). In the subsequent plasma experiment, the breakdown voltage in the discharge was reduced from 7 to $5 \mathrm{~V}$ as compared to the Globus-M tokamak. The result indirectly indicates a higher quality of manufacture and assembly of the Globus-M2 electromagnetic system. The first plasma in Globus-M2 was achieved on 23 April 2018. During the first campaign, moderate density plasma shot with a current of $200 \mathrm{kA}$ was reproduced (see figure 25), and a $0.6 \mathrm{~T}$ toroidal magnetic field was achieved. A full-scale experiment with an increased toroidal magnetic field is planned for the beginning of 2019.

\section{Summary}

The main results of the recent tokamak-related research at the Ioffe Institute have been reviewed. Major parts of the studies were conducted at three small tokamaks with different parameters-the spherical tokamak Globus-M, the large aspect ratio tokamak FT-2 and the compact tokamak TUMAN-3M. The main fields of study were energy confinement (Globus-M and FT-2); the $\mathrm{L}-\mathrm{H}$ transition (TUMAN-3M and FT-2); Alfvén waves (Globus-M and TUMAN-3M); ion cyclotron emission (TUMAN-3M); major plasma discharge disruption (Globus-M) and the SOL (Globus-M). Benchmarking of the full-f global gyrokinetic modeling results against the DR data using synthetic diagnostics was performed at the FT-2 tokamak. Anomalous emission and absorption due to parametric excitation of localized UH waves in ECRH experiments was also discussed. Three ITER diagnostics are described: NPA, gamma-ray spectrometry and DTS combined with LIF. A majority of the future studies will be concentrated around the recently launched Globus-M2 spherical tokamak. The machine has an electromagnetic system, providing a $1 \mathrm{~T}$ toroidal magnetic field, upgraded diagnostics and heating systems. Globus-M2 is considered as the next step on the path to a compact beam-plasma neutron source due to its distinguishing features such as a high normalized Larmor radius and reactor-scale heating power density (up to $6 \mathrm{MW} \mathrm{m}^{-3}$ ). It will provide relevant conditions for the studies of new tokamak physics and the testing of new fusion technologies.

\section{Acknowledgment}

The experimental investigations were performed on the Unique Scientific Facility 'Spherical Tokamak Globus-M', which is incorporated in the Federal Joint Research Center 'Material Science and Characterization in Advanced Technology', on the Unique Scientific Facility 'TUMAN-3M' and on the FT-2 tokamak. The maintenance of tokamaks and of standard diagnostics was supported by the Ioffe Institute. Auxiliary heating and fast particle confinement studies in section 2.1 were supported by RSF research project No. 17-72-20076. TAE investigation (section 4.1) was supported by RSF research project No. 17-12-01177. Studies of Alfven waves and ICE (sections 4.2 and 5) on the TUMAN-3M tokamak were supported by RSF grant 16-12-10285. Investigations presented in sections 2.2 and 8 were performed under financial support of the RSF grant 17-12-01110. Investigations presented in section 9 of the paper were performed under financial support of the RSF grant 16-12-10043.

\section{ORCID iDs}

A.B. Altukhov (10 https://orcid.org/0000-0002-5913-6004 N.A. Babinov (1) https://orcid.org/0000-0002-8695-2701 A.A. Belokurov (1) https://orcid.org/0000-0001-8942-6734

V.V. Bulanin (1) https://orcid.org/0000-0001-8030-3957

A.D. Gurchenko (1) https://orcid.org/0000-0002-2540-2511

T.P. Kiviniemi (1) https://orcid.org/0000-0001-9753-3388

S.V. Lebedev (1) https://orcid.org/0000-0001-5979-5344

C. Lechte (1) https://orcid.org/0000-0003-4171-888X

P. Niskala @ https://orcid.org/0000-0001-7308-9921

A.Yu. Popov (1) https://orcid.org/0000-0003-0574-516X

A.Yu. Telnova (1) https://orcid.org/0000-0003-0495-7881

L.A. Varshavchick (1) https://orcid.org/0000-0002-7042-3469 


\section{References}

[1] Gusev V.K. et al 2013 Nucl. Fusion 53093013

[2] Gusev V.K. et al 2015 Plasma Phys. Control. Fusion 58014032

[3] Winsor N., Johnson J.L. and Dawson J.M. 1968 Phys. Fluids 112448

[4] Lashkul S.I. et al 2017 EPJ Web Conf. 14903012

[5] Valovic M. et al 2009 Nucl. Fusion 49075016

[6] Kaye S.M. et al 2006 Nucl. Fusion 46 848-57

[7] ITER Physics Basis Expert Group Chapter 2: Plasma confinement and transport 1999 Nucl. Fusion 392175

[8] Valovic M. et al 2011 Nucl. Fusion 51073045

[9] Kaye S.M. et al 2013 Nucl. Fusion 53063005

[10] Kurskiev G.S. et al 2017 Phys. At. Nuclei 801313

[11] Kurskiev G.S. et al 2017 Plasma Phys. Control. Fusion 59045010

[12] Kurskiev G.S. et al 2019 Nucl. Fusion 59066032

[13] Telnova A.Yu. et al 2017 J. Phys.: Conf. Ser. 907012014

[14] Kouprienko D.V. et al 2018 45th EPS Conf. on Plasma Phys. (Proc. of 45th EPS Conf. on Plasma Phys.) vol 42A (Prague) (ECA) p P4.1097 (http://ocs.ciemat.es/ EPS2018PAP/pdf/P4.1097.pdf)

[15] Simmet E.E. and The ASDEX Team 1996 Plasma Phys. Control. Fusion 38 689-704

[16] Staebler G.M. 1998 Plasma Phys. Control. Fusion 40 569-80

[17] Malkov M. and Diamond P. 2008 Phys. Plasmas 15122301

[18] Askinazi L.G. et al 2017 Plasma Phys. Control. Fusion 59014037

[19] Belokurov A.A. et al 2018 Nucl. Fusion 58112007

[20] Petrov Yu.V. et al 2014 Tech. Phys. Lett. 40 1136-9

[21] Petrov Yu. V. et al 2015 JPP 81515810601

[22] Bakharev N.N. et al 2018 Nucl. Fusion 58126029

[23] Bakharev N.N. et al 2013 Tech. Phys .Lett. 391085

[24] Yashin A.Y. et al 2015 J. Instrum. 1010023

[25] Bulanin V.V. et al 2017 Tech. Phys. Lett. 43 1067-70

[26] Gusev V.K. et al 2018 Tech. Phys. Lett. 44 67-9

[27] Askinazi L.G. et al 2015 Nucl. Fusion 55104013

[28] Abdullina G.I. et al 2018 Tech. Phys. Lett. 44108

[29] Abdullina G.I. et al 2018 J. Phys.: Conf. Ser. 1094012008

[30] Lebedev S.V. et al 2016 43rd EPS Conf. on Plasma Physics (Proc. of 43rd EPS Conf. on Plasma Phys.) vol 41F (ECA) (Leuven) p P5.036 (http://ocs.ciemat.es/EPS2016PAP/pdf/ P5.036.pdf)

[31] Abdullina G.I. et al 2018 45th EPS Conf. on Plasma Physics (Proc. of 45th EPS Conf. on Plasma Phys.) (Prague) $\mathrm{p}$ P4.1062 (http://ocs.ciemat.es/EPS2018PAP/pdf/P4.1062.pdf)

[32] McClements K.G. et al 2002 Nucl. Fusion 421155

[33] Tukachinsky A.S. et al 2016 Tech. Phys. Lett. 421167

[34] Cottrell G.A. 2000 Phys. Rev. Lett. 842397

[35] Askinazi L.G. et al 2018 Nucl. Fusion 58082003

[36] Lebedev S.V. et al 2017 EPJ Web Conf. 14903010

[37] Ochoukov R. et al 2019 Nucl. Fusion 59014001

[38] Rome J.A. and Peng Y.-K.M. 1979 Nucl. Fusion 191193

[39] Sharapov S.E. et al 2014 Phys. Plasmas 21082501

[40] Mikhailovsky A. 1971 Nucl. Fusion 11323

[41] Eidietis N.W. et al 2015 Nucl. Fusion $\mathbf{5 5} 063030$

[42] Sakharov N.V. et al 2017 Plasma Phys. Rep. 43422

[43] Sakharov N.V. et al 2018 Plasma Phys. Rep. $44335-46$

[44] Tokarev V.A. et al 2018 J. Phys.: Conf. Series 1094012003

[45] Eich T. et al 2011 Phys. Rev. Lett. 107215001

[46] Stangeby P.C. 2000 The Plasma Boundary of Magnetic Fusion Devices (Bristol: IOP Publishing Ltd)

[47] Eich T. et al 2013 Nucl. Fusion 53093031

[48] Gusev V.K. et al 2015 Nucl. Fusion 55104016
[49] Krutkin O. et al 2019 Nucl. Fusion 59096017

[50] Altukhov A.B. et al 2018 Phys. Plasmas 25082305

[51] Lechte C. et al 2017 Plasma Phys. Control. Fusion 5907500

[52] Gusakov E.Z. and Popov A.Yu. 2002 Plasma Phys. Control. Fusion 442327

[53] Gusakov E.Z. and Popov A.Yu. 2005 Plasma Phys. Control. Fusion 47379

[54] Cohen B. et al 1991 Rev. Mod. Phys. 63949

[55] Westerhof E. et al 2009 Phys. Rev. Lett. 103125001

[56] Gusakov E.Z. and Popov A.Yu. 2016 Phys. Plasmas 23082503

[57] Popov A.Yu. and Gusakov E.Z. 2016 Europhys. Lett. 11645002

[58] Gusakov E.Z. et al 2018 Preprint: 2018 IAEA Fusion Energy Conf. (Gandhinagar, India, 22-27 October 2018) TH/P4-10 (https://nucleus.iaea.org/sites/fusionportal/Shared\%20 Documents/FEC\%202018/fec2018-preprints/preprint0057. pdf)

[59] Afanasyev V.I. et al 2016 30th Meeting of the ITPA TGD (Novosibirsk, Russian Federation, 21-24 June 2016)

[60] Petrov S.Ya. et al 2017 Phys. At. Nuclei 80 1268-78

[61] Afanasyev V.I. et al 2010 Nucl. Instrum. Methods Phys. Res. A $621456-67$

[62] Afanasyev V.I. et al 2015 Plasma Phys. Rep. 41 983-89

[63] Petrov M.P. et al 2015 1st EPS Conf. on Plasma Diagnostics (Frascati, Italy, 14-17 April 2015) p 153 (https://pos.sissa. it/240/153/pdf)

[64] Mironov M. et al 2018 Nucl. Fusion 58082030

[65] Gin D. et al 2014 AIP Conf. Proc. 1612149

[66] Gin D. et al 2018 Preprint: 2018 IAEA Fusion Energy Conf. (Gandhinagar, India, 22-27 October 2018) FIP/P7-15 (https://nucleus.iaea.org/sites/fusionportal/Shared\%20 Documents/FEC\%202018/fec2018-preprints/preprint0479. pdf)

[67] Shevelev A.E. et al 2013 Nucl. Fusion 53123004

[68] Shevelev A.E. et al 2016 Nucl. Instrum. Methods Phys. Res. A $830102-8$

[69] Shevelev A.E. et al 2018 Nucl. Fusion 58016034

[70] Shevelev A.E. et al 2018 Plasma Phys. Control. Fusion 600750097

[71] Reux C. et al 2015 Nucl. Fusion 55093013

[72] Nocente M. et al 2018 Rev. Sci. Instrum. 89101124

[73] Schneider M. et al 2016 Nucl. Fusion 5611202210

[74] Mukhin E.E. et al 2014 Nucl. Fusion 54043007

[75] Mukhin E.E. et al 2019 Nucl. Fusion 59086052

[76] Gorbunov A. et al 2017 Fusion Eng. Des. 123695

[77] Mukhin E.E. et al 2017 Fusion Eng. Des. 123686

[78] Kornev A. et al 2018 SOFT (Messina, Sicily, 16-21 September 2018) p P2.055 (www.soft2018.eu/?download=Book-ofAbstracts.pdf)

[79] Razdobarin A. 2018 SOFT (Messina, Sicily, 16-21 September 2018) p P4.215 (www.soft2018.eu/?download=Book-ofAbstracts.pdf)

[80] Minaev V.B. et al 2017 Nucl. Fusion 57066047

[81] Bakharev N.N. et al 2015 Nucl. Fusion 55043023

[82] Saveliev A. et al 2011 38th EPS Conf. on Plasma Phys. (Proc. of 38th EPS Conf. on Plasma Phys.) vol 35 (ECA) (Strasbourg) p GP-4.103 (http://ocs.ciemat.es/ EPS2011PAP/pdf/P4.103.pdf)

[83] Shcherbinin O. et al 2012 Tech. Phys. Lett. 38 869-72

[84] Shchegolev P.B. et al 2015 Tech. Phys. 601321

[85] Minaev V.B. et al 2018 45th EPS Conf. on Plasma Phys. (Proc. of 45th EPS Conf. on Plasma Phys.) vol 42A (ECA) (Prague) p P4.1065 (http://ocs.ciemat.es/EPS2018PAP/pdf/ P4.1065.pdf)

[86] Minaev V.B. et al 2018 J. Phys.: Conf. Ser. 1094012001 